Supporting Information

\title{
Exo-selective, Reductive Heck Derived Polynorbornenes with Enhanced Molecular Weights, Yields, and Hydrocarbon Gas Transport Properties
}

Benjamin J. Sundell, ${ }^{*}$ John A. Lawrence III,* Daniel J. Harrigan, Sibo Lin, Tatiana P. Headrick, Jeremy T. O’Brien, William F. Penniman, Nathan Sandler

Aramco Services Company: Aramco Research Center - Boston, Massachusetts, 02139

\section{Table of Contents}

Experimental section (XRD, FTIR, TGA, EA, membrane formation, NMR, GPC, pure gas permeation measurements, mixed gas permeation measurements, monomer synthesis, polymer synthesis, computational protocol)

Synthetic mechanism for formation of norbornene and tricyclononene monomers

Monomer synthesis table (monomer, catalyst used, solvent, time, temp, yield)S9

Polymer synthesis table (monomer, catalyst system, solvent, time, temperature, yield, Mn, $\mathrm{Mn} / \mathrm{Mw})$ S11

XRD spectra of individual polymers S12

FTIR spectra of polymers S13

TGA of polymers S14

${ }^{1} \mathrm{H}$ NMR of monomers and polymers S16

Elemental analysis of polymers S2O

GPC chromatograms of polymers S2O

Computational modeling of APTCN-exo-BPTMS compared to APTCN-endo-BPTMS 


\section{Experimental}

Interchain spacing within each polymeric sample was determined through X-ray diffraction (XRD), measured using a Bruker D8 Discover diffractometer. Monochromatic radiation was emitted through a copper tube at $1.5418 \AA$, passed through a $0.3 \mathrm{~mm}$ collimator and micromask, and recorded by a VANTEC-500 2-D detector. A metal plate was placed above the sample during each scan to reduce air scattering and background noise. Scanning was performed with $2 \theta$ ranging from $10-55^{\circ}$ at $15^{\circ}$ intervals. Post-processing and analysis was performed with Diffrac.Eva 3.0 software. The interchain spacing, also referred to as $d$ spacing, is correlated to the peak scattering angles through Bragg's Law, denoted as:

$$
d_{\text {Bragg's }}=\frac{\lambda}{2 \sin \theta}
$$

Fourier transform infrared spectroscopy (FTIR) spectra were collected and analyzed with a Thermo Scientific Nicolet IS5oR system with attenuated total reflectance (ATR) capabilities and OMNIC software. Scans of atmospheric conditions for background correction were taken prior to sample loading onto the aperture, and the spectra were obtained with 128 scans at a resolution of $4 \mathrm{~cm}^{-1}$.

Thermogravimetric analysis (TGA) was performed with a TA Discovery Series ${ }^{\mathrm{TM}}$ TGA. Testing of the PNB samples was executed at a heat rate of $10^{\circ} \mathrm{C} \mathrm{min}{ }^{-1}$ up to $700{ }^{\circ} \mathrm{C}$ under nitrogen gas. Prior to experimentation, all samples were heat treated within an Isotemp ${ }^{\oplus}$ vacuum oven at a constant $100{ }^{\circ} \mathrm{C}$ overnight to remove excess moisture or residual solvent post-polymerization.

Elemental analysis was performed by Midwest Microlab, using combustion analysis for carbon, oxygen, and hydrogen. Complete combustion was achieved using a Sn capsule and combustion aid.

For polymeric membrane formation, 0.5-0.6 grams of each sample were stirred until dissolved in $10 \mathrm{~mL}$ of toluene. The polymer solutions were filtered using $1 \mu \mathrm{m}$ PTFE syringe filters, and then casted into clean $10 \mathrm{~cm}$ diameter perfluoroalkoxy alkane (PFA) molds on a level surface, covered with glass Pyrex ${ }^{\circledR}$ dishes to control the evaporation of toluene, and allowed to dry overnight. Samples were then put into the vacuum oven at $110{ }^{\circ} \mathrm{C}$ for 24 hours to remove any trace solvent. Polymer thickness is controlled by the solution concentration, with a desired range between 50-100 $\mu \mathrm{m}$.

Nuclear magnetic resonance (NMR) spectroscopy was performed using a Bruker Ascend ${ }^{\mathrm{TM}}$ 500 spectrometer utilizing TopSpin ${ }^{\mathrm{TM}}$ 3.2. $1 \mathrm{H}-\mathrm{NMR}$ spectra were collected at $500 \mathrm{MHz}$ and 13C-NMR spectra were collected at $125 \mathrm{MHz}$. All spectra were obtained from 1-5\% (w/v) 0.7 $\mathrm{mL}$ solutions in $\mathrm{CDCl}_{3}$. 
Gel permeation chromatography (GPC) system consisted of an integrated pump (Malvern GPCmax, Malvern Instruments, Houston, TX) that included an online degasser, autosampler, and heated sample tray. A Malvern T40oo styrene-divinyl benzene singlepore column (300x8mm) connected in series with a TGuard guard column was used as a stationary phase. A system of multiple detectors (Malvern TDAmax, Malvern Instruments, Houston, TX) connected in series was used for analysis. Low angle and right angle light scattering detectors (LALS and RALS) operating at $670 \mathrm{~nm}$, a viscometer detector, a refractive index detector, and a UV detector (Malvern 302/305 UV-PDA Detector Model 260o, Malvern Instruments, Houston, TX) with a deuterium light source operating between 190 and $500 \mathrm{~nm}$ provided online results. The system was corrected for interdetector delay, band broadening, and light scattering signal variation using a 104,401 $\mathrm{g} / \mathrm{mol}$ polystyrene standard (Malvern). Data acquisition and analysis was conducted using OMNISEC software. The mobile phase was HPLC grade tetrahydrofuran (Fisher). The sample solutions were prepared in the concentration range of $1-2 \mathrm{mg} / \mathrm{mL}$ and were filtered using a $0.2 \mu \mathrm{m}$ PTFE filters. Molecular weight values were measured using online light scattering and differential refractive index signals.

Gas permeation was conducted using a constant volume, variable pressure technique. The downstream side of the membrane was maintained at less than 25 torr using a vacuum pump. To keep the feed composition constant, a retentate stream was applied. The retentate flow was adjusted to 100 times the permeate flow. Composition of the permeate stream was measured using a Shimadzu GC equipped with a thermal conductivity detector. A pump (Teledyne Isco) was used to control the feed pressure. The feed gas was heated to $32^{\circ} \mathrm{C}$ to maintain the feed above its hydrocarbon dew point of $\sim 21^{\circ} \mathrm{C}$ at 800 psi. The membrane cell was kept at room temperature. Multiple membrane samples were prepared from the same starting film, and standard deviations of permeability and selectivity were reported by taking the average of at least three samples for pure gas permeation testing and two samples for mixed gas permeation testing.

Permeability was calculated using Equation 1 , where $y_{i}$ is the downstream mole fraction, $l$ the membrane thickness and $\Delta f_{i}$ the partial fugacity difference across the membrane. A fugacity driving force was used rather than partial pressure to correct for gas phase nonidealities. Fugacity coefficients were calculated using the Peng Robinson equation of state. The steady-state flux, or flux total, was calculated by measuring the downstream pressure rise $\left(\frac{d p}{d t}\right)$ after 30 minutes of permeation time. This time was well beyond the steady-state time estimated from 14x the pure gas time lag, which equated to $\sim 10$ minutes for the slowest gas, methane. At 800 psi, the permeability was measured after 1 hour of permeation time to allow for any swelling induced relaxations to set-in. 
(2) $P_{i}=\frac{\text { flux }_{\text {total }} \cdot y_{i} \cdot l}{\Delta f_{i}}$

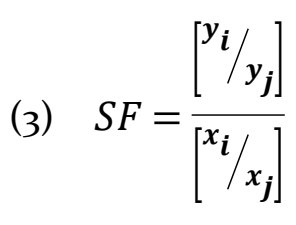

(4) $\quad \alpha_{\mathrm{f}}=P_{i} / P_{j}$

Fugacity selectivity (Equation 4) may be obtained by taking the ratio of gas permeabilities described in Equation 2. A schematic of the gas permeation cell is shown in Figure S1.

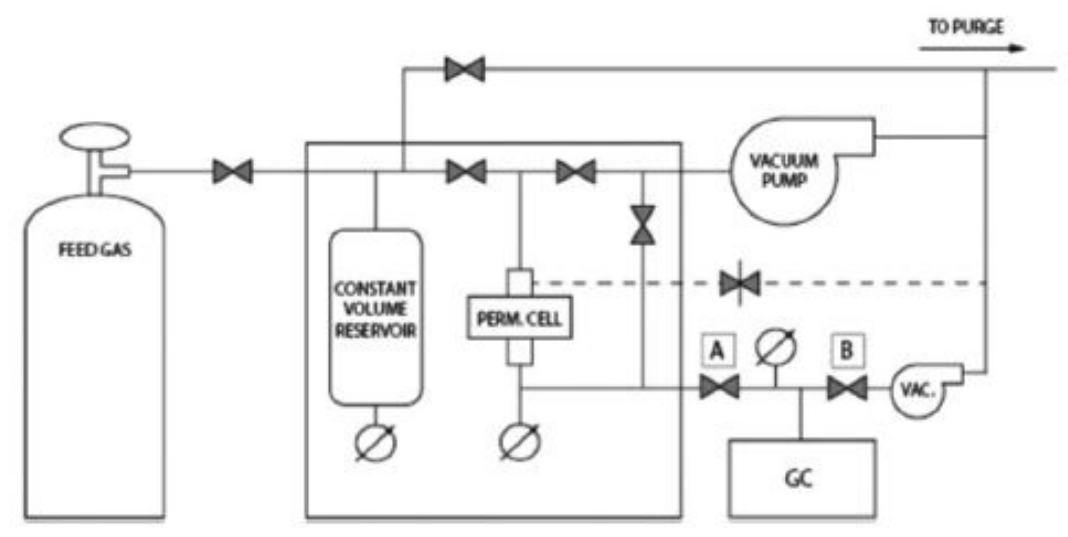

Figure S1. Gas permeation cell used for pure and mixed gas experimentation

Each norbornene monomer was constructed, hydrogenated, and minimized with Avogadro. ${ }^{1}$ The atomic numbering was adjusted such that the capping hydrogen atoms representing the head and tail directions of the polymer backbone were set as the first and last atoms in the molecular coordinates file. Using the hydrogenated monomer coordinates file and custom python code built on the PySimm² framework, the syndiotactic-(exo,exo) $\mathrm{H}$-terminated 20-mer was constructed using rigid mathematical translation, rotation, and reflection operations. From this strained oligomer, the remaining operations were then conducted with PySimm using Gasteiger atomic charges ${ }^{3}$ and the Dreiding forcefield. 4

(1) The oligomer was optimized using the Fast Inertial Relaxation Engine (FIRE) as implemented in LAMMPS. 5

(2) Eight copies of the FIRE-optimized oligomer were randomly inserted into a dilute simulation box $(\rho=0.005)$ to ensure no hardcore overlaps were introduced into the system.

(3) The eight 20-mers were then computationally annealed using the 21-step equilibration scheme of the Colina group $6\left(\mathrm{P}_{\max }=50000\right.$ bar $)$ and LAMMPS. 
The equilibrated systems were analyzed with Poreblazer7 to obtain system densities. The void spaces of the systems were visualized using $\mathrm{Jmol}^{8}$ with a probe radius of 2.15 Angstroms to represent butane ${ }^{9}$ (Jmol command: isosurface cavity 2.15). Fractional free volume (FFV) was determined via the Bondi method ${ }^{10}$ :

$$
\mathrm{FFV}=1-1.3^{*} \mathrm{~V}_{\mathrm{vdw}} / \mathrm{V}_{\mathrm{sp}}
$$

where $\mathrm{V}_{\mathrm{sp}}$, the specific volume, and $\mathrm{V}_{\mathrm{vdw}}$, the occupied volume, were derived from Poreblazer output:

$$
\begin{gathered}
\mathrm{V}_{\mathrm{sp}}=\text { "System volume" } \\
\mathrm{V}_{\mathrm{vdw}}=\text { "System volume" - "Geometric (point accessible) volume" }
\end{gathered}
$$

For each monomer, three independent simulations of eight 20-mers were equilibrated to obtain average values and standard deviations for FFV and system density.

\section{References}

1. Hanwell, M. D.; Curtis, D. E.; Lonie, D. C.; Vandermeersch, T.; Zurek, E.; Hutchison, G. R., Avogadro: an advanced semantic chemical editor, visualization, and analysis platform. Journal of Cheminformatics 2012, 4 (1), 17.

2. Fortunato, M. E.; Colina, C. M., pysimm: A python package for simulation of molecular systems. SoftwareX 2017, 6, 7-12.

3. Gasteiger, J.; Marsili, M. Tetrahedron Letters 1978, 19, 3181.

4. Mayo, S. L.; Olafson, B. D.; Goddard, W. A. The Journal of Physical Chemistry 1990, 94, 8897.

5. Plimpton, S., Fast Parallel Algorithms for Short-Range Molecular Dynamics. Journal of Computational Physics 1995, 117 (1), 1-19.

6. Abbott, L. J.; Hart, K. E.; Colina, C. M., Polymatic: A generalized simulated polymerization algorithm for amorphous polymers. Theor Chem Acc 132.

7. Sarkisov, L.; Harrison, A., Computational structure characterisation tools in application to ordered and disordered porous materials. Molecular Simulation 2011, 37 (15), 1248-1257.

8. Jmol: an open-source Java viewer for chemical structures in $3 \mathrm{D}$. http://www.jmol.org.

9. McKeen, L. W., 1 - Introduction to Permeation of Plastics and Elastomers. In Permeability Properties of Plastics and Elastomers (Third Edition), McKeen, L. W., Ed. William Andrew Publishing: Oxford, 2012; pp 1-20. 
10. Van Krevelen, D. W.; Te Nijenhuis, K., Properties of polymers: their correlation with chemical structure; their numerical estimation and prediction from additive group contributions. Elsevier: 2009.

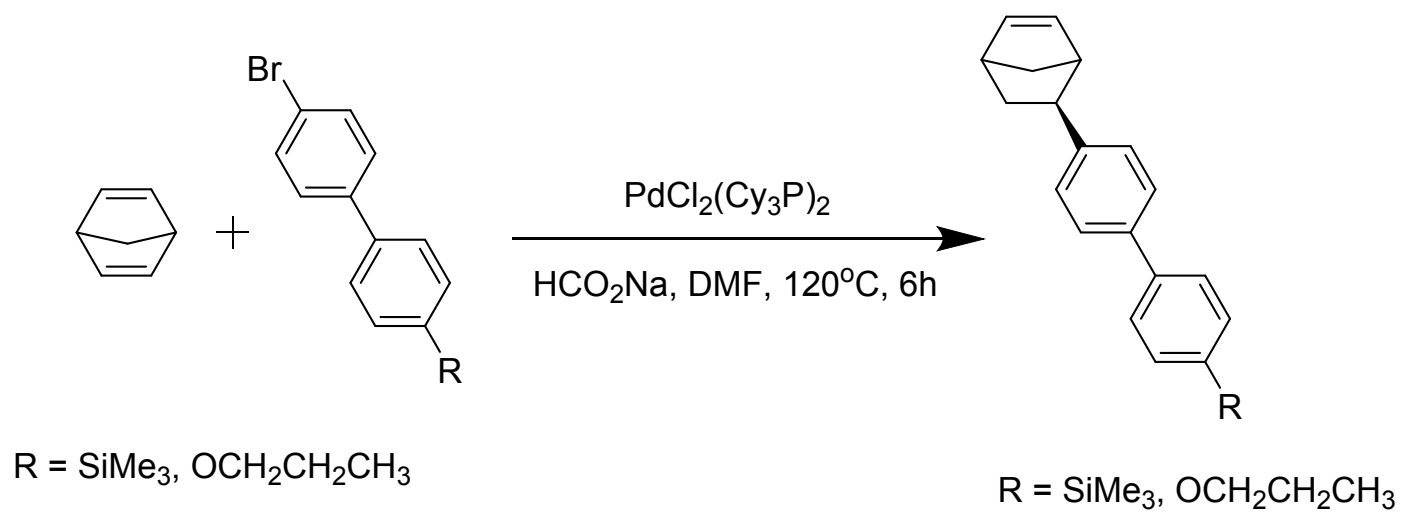

Scheme S1. Synthesis of Mizoroki-Heck derived norbornene monomers

Representative synthesis: A dried 1 L round bottom flask was equipped with a stir bar, cooled to room temperature and evacuated under vacuum. The flask was charged with nitrogen, and $\mathrm{PdCl}_{2}\left(\mathrm{PCy}_{3}\right)_{2}$ (5.112 g, $\left.7 \mathrm{mmol}\right), \mathrm{NaHCO}_{3}(23.355 \mathrm{~g}, 343 \mathrm{mmol})$, and aryl bromide ( $20 \mathrm{~g}, 69 \mathrm{mmol}$ ) were added under an inert atmosphere. $300 \mathrm{~mL}$ of anhydrous DMF was added via syringe followed by norbornadiene $(34.926 \mathrm{~mL}, 343 \mathrm{~mol})$. The reaction was then stirred at $120{ }^{\circ} \mathrm{C}$ for six hours. The entire reaction mixture was added to $150 \mathrm{~mL}$ of water and diluted with $150 \mathrm{~mL}$ of diethyl ether. The aqueous layer was washed three times with diethyl ether, and the collected diethyl ether layers were washed with a fresh $100 \mathrm{~mL}$ of DI water. The organic layer was collected over $\mathrm{MgSO}_{4}$ and then filtered. Diethyl ether was removed under vacuum to afford a viscous residue as the crude product, which was then purified with column chromatography using $100 \%$ hexanes as an eluent.

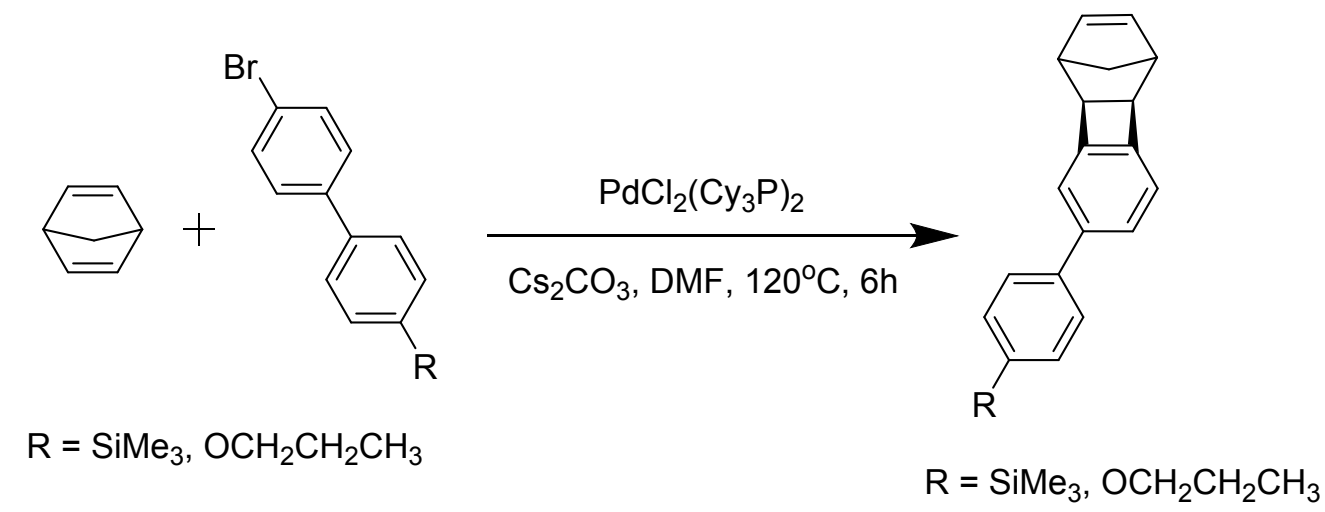

Scheme S2. Synthesis of Mizoroki-Heck derived tricyclononene monomers

Representative synthesis: A 100-mL Schlenk flask was equipped with a stir bar, flame dried with a butane torch, cooled to room temperature and evacuated under vacuum. The flask was charged with nitrogen, and $\mathrm{PdCl}_{2}\left(\mathrm{PCy}_{3}\right)_{2}(0.375 \mathrm{~g}, 0.508 \mathrm{mmol}), \mathrm{Cs}_{2} \mathrm{CO}_{3}$ (9.220 g, 28.210 $\mathrm{mmol})$, and aryl bromide $(4.306 \mathrm{~g}, 14.105 \mathrm{mmol})$ were added under an inert atmosphere. 25 
$\mathrm{mL}$ of DMF was added via syringe, followed by norbornadiene $(5.738 \mathrm{~mL}, 56.421 \mathrm{mmol})$. The reaction was then stirred at $120^{\circ} \mathrm{C}$ for six hours. The entire reaction mixture was added to $150 \mathrm{~mL}$ of water and diluted with $150 \mathrm{~mL}$ of diethyl ether. The aqueous layer was washed three times with diethyl ether, and the collected diethyl ether layers were washed with a fresh $100 \mathrm{~mL}$ of DI water. The organic layer was collected over $\mathrm{MgSO}_{4}$ and then filtered. Diethyl ether was removed under vacuum to afford a brown viscous residue as the crude product, which was then purified with column chromatography using $100 \%$ hexanes as an eluent.

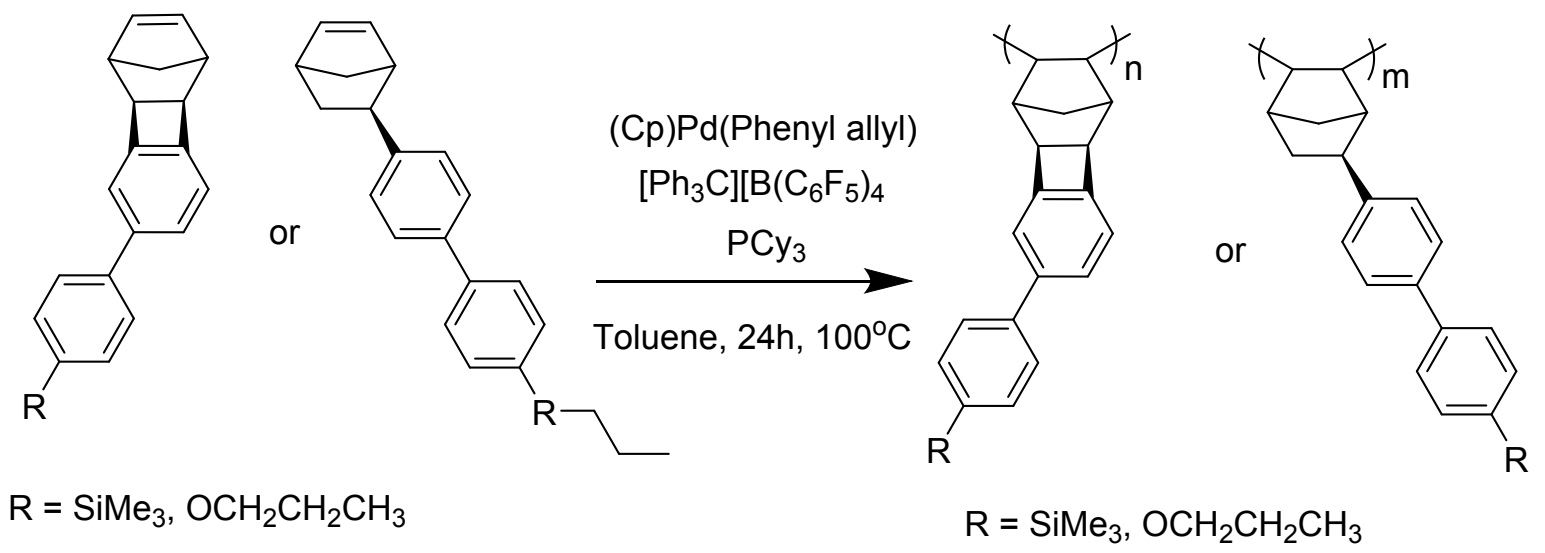

Scheme $S_{3}$. Synthesis of addition-type norbornene and tricyclononene polymers

Representative synthesis: Under nitrogen, a $40 \mathrm{~mL}$ vial was charged with tricyclononene monomer (o.50 g, $1.65 \mathrm{mmol}$ ) and $30 \mathrm{~mL}$ of dry, deoxygenated toluene. In three separate vials, tricyclohexylphosphine (o.093 mg, 0.00033 mmol), cyclopentadienyl-(1,2,3-n)-1phenyl-2-propenyl palladium (II) (0.095 $\mathrm{mg}, 0.00033 \mathrm{mmol}$ ), and trityl tetrakis [3,5bis(trifluoromethyl)phenyl]borate $(0.305 \mathrm{mg}, 0.00033 \mathrm{mmol}$ ) were each dissolved in toluene. The palladium catalyst was thoroughly mixed with the phosphine solution, and then the trityl borate solution was added to the palladium and phosphine mixture. Afterwards, the mixed catalyst-phosphine-activator solution was added to the monomer solution. The reaction vessel was sealed and brought out of the glovebox to heat and stir at $100{ }^{\circ} \mathrm{C}$ for 24 hours, where it became viscous. After 24 hours, the solution was precipitated in $1000 \mathrm{~mL}$ of acetone dropwise which immediately formed small white polymer beads stirring in the acetone and dissolved any residual starting monomer. The white polymer was stirred overnight, collected via filtration, and dried in vacuo to constant weight. No evidence of starting material was observed via ${ }^{1} \mathrm{H}-\mathrm{NMR}$ (no olefinic peaks detected in the $\delta$ 6.o - 6.5 ppm region) or GPC. 

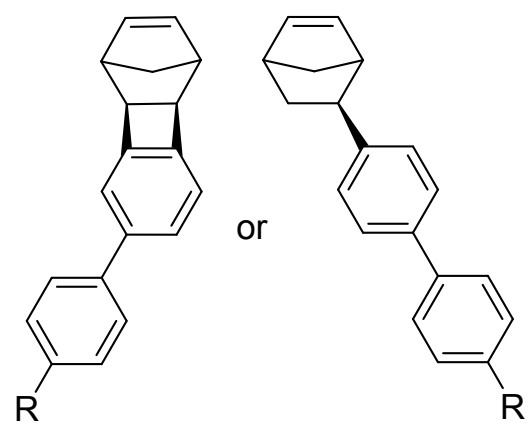

$$
\mathrm{R}=\mathrm{SiMe}_{3}, \mathrm{OCH}_{2} \mathrm{CH}_{2} \mathrm{CH}_{3}
$$
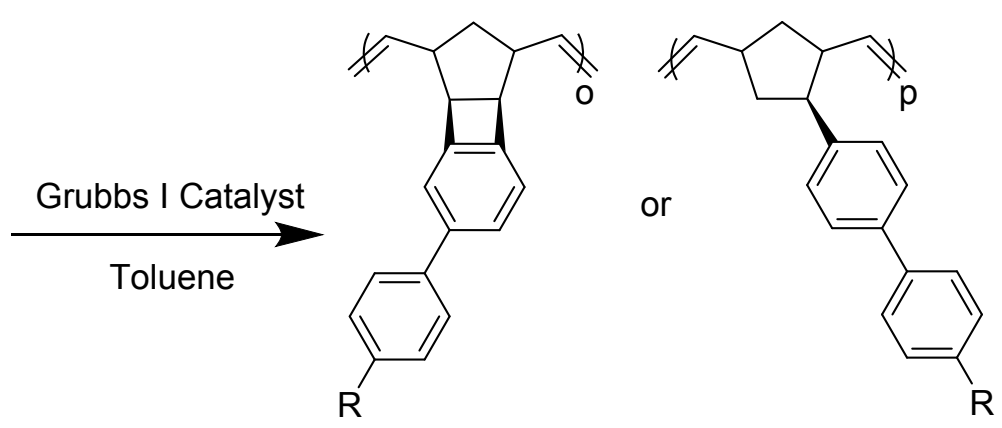

$\mathrm{R}=\mathrm{SiMe}_{3}, \mathrm{OCH}_{2} \mathrm{CH}_{2} \mathrm{CH}_{3}$

Scheme $\mathbf{S}$. Synthesis of ROMP norbornene and tricyclononene polymers

Representative synthesis: In a glovebox, a $30 \mathrm{~mL}$ vial was charged with tricyclononene monomer (0.500 g, $1.65 \mathrm{mmol}$ ) and $25 \mathrm{~mL}$ of dry, deoxygenated toluene. In a separate vial, Grubbs first generation catalyst $(13.6 \mathrm{mg}$, $0.0165 \mathrm{mmol}$ ) was dissolved in $1 \mathrm{~mL}$ of dry toluene to create a stock catalyst solution. Finally, $0.1 \mathrm{~mL}$ of the catalyst solution was added to the stirring monomer solution to initiate polymerization. After $24 \mathrm{~h}$, ethyl vinyl ether ( $0.48 \mathrm{~mL}$, $5 \mathrm{mmol}$ ) was added to terminate the polymerization, and the solution continued to stir. After another 30 min viscous solution was precipitated dropwise into stirring acetone (1000 $\mathrm{mL}$ ). The white polymer was collected via filtration and dried in vacuo to constant weight.

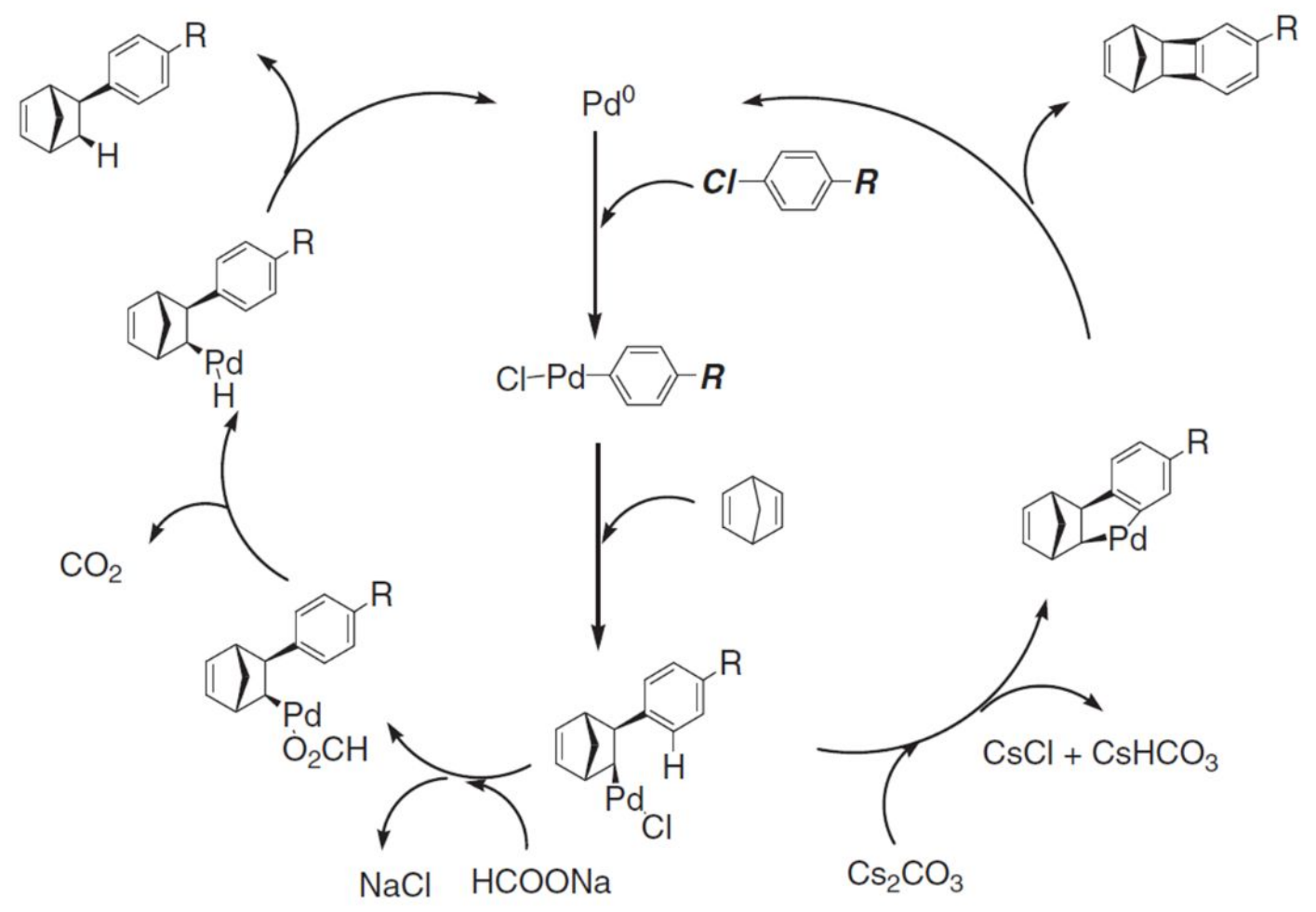

Figure S2. Hypothesized mechanism reproduced from Aida et al. ${ }^{1}$ The left side of this mechanism utilizes sodium formate as a reducing agent to achieve norbornene type 
monomer, whereas the right side uses cesium carbonate in the absence of a reducing agent to yield tricyclononene type monomer

Table S1. Monomer syntheses as a function of monomer type, catalyst system, stoichiometry, and reaction conditions. Table also includes norbornene structures that, due to lack of monomer reaction or purity, resulted in starting materials not suitable for polymerization

\begin{tabular}{|c|c|c|c|c|c|c|c|c|c|}
\hline Structure & substituent & $\begin{array}{l}\text { catalyst } \\
\text { system }\end{array}$ & solvent & $\begin{array}{l}\text { NBD } \\
\text { mol } \\
\text { eq. }\end{array}$ & $\begin{array}{l}\text { catalyst } \\
\text { mol eq. }\end{array}$ & $\begin{array}{l}\text { co- } \\
\text { catalyst } \\
\text { mol eq. }\end{array}$ & $\begin{array}{l}\text { Time } \\
(\mathbf{h r})\end{array}$ & $\begin{array}{l}\text { Temp } \\
\left({ }^{\circ} \mathrm{C}\right)\end{array}$ & $\begin{array}{l}\text { Yield } \\
(\%)\end{array}$ \\
\hline $\begin{array}{l}\text { norbornene } \\
\left(4,5^{-}\right. \\
\text {substituted })\end{array}$ & $\begin{array}{l}\text { 4:-Ph } \\
\text { 5:-BPP }\end{array}$ & $\begin{array}{l}\mathrm{Pd}\left(\mathrm{PPh}_{3}\right)_{4} \\
\mathrm{Na}^{+} \mathrm{Ph}_{4} \mathrm{~B}^{-}\end{array}$ & anisole & 4.0 & 0.018 & 2 & 20 & 120 & - \\
\hline $\begin{array}{l}\text { norbornene } \\
\left(4,5^{-}\right. \\
\text {substituted) }\end{array}$ & $\begin{array}{l}\text { 4:-Ph } \\
\text { 5:-BPP }\end{array}$ & $\begin{array}{l}\mathrm{Pd}\left(\mathrm{PPh}_{3}\right)_{4} \\
\mathrm{Na}^{+} \mathrm{Ph}_{4} \mathrm{~B}^{-}\end{array}$ & DMF & 4.0 & 0.018 & 2.0 & 6 & 120 & - \\
\hline $\begin{array}{l}\text { norbornene } \\
\left(4,5^{-}\right. \\
\text {substituted) }\end{array}$ & $\begin{array}{l}\text { 4:-Ph } \\
\text { 5:-BPP }\end{array}$ & $\begin{array}{l}\mathrm{Pd}\left(\mathrm{PPh}_{3}\right)_{4} \\
\mathrm{Na}^{+} \mathrm{Ph}_{4} \mathrm{~B}^{-}\end{array}$ & DMF & 4.0 & 0.018 & 2.0 & 6 & 100 & - \\
\hline $\begin{array}{l}\text { norbornene } \\
\left(4,5^{-}\right. \\
\text {substituted) }\end{array}$ & $\begin{array}{l}\text { 4: -Ph } \\
\text { 5: -BPP }\end{array}$ & $\begin{array}{l}\mathrm{Pd}\left(\mathrm{PPh}_{3}\right)_{4} \\
\mathrm{Na}^{+} \mathrm{Ph}_{4} \mathrm{~B}^{-}\end{array}$ & DMSO & 4.0 & 0.018 & 2.0 & 6 & 100 & - \\
\hline $\begin{array}{l}\text { norbornene } \\
\left(4,5^{-}\right. \\
\text {substituted) }\end{array}$ & $\begin{array}{l}\text { 4:-Ph } \\
\text { 5:-BPP }\end{array}$ & $\begin{array}{l}\mathrm{Pd}\left(\mathrm{PPh}_{3}\right)_{4} \\
\mathrm{Na}^{+} \mathrm{Ph}_{4} \mathrm{~B}^{-}\end{array}$ & DMAc & 4.0 & 0.018 & 2.0 & 20 & 100 & - \\
\hline $\begin{array}{l}\text { norbornene } \\
\left(4,5^{-}\right. \\
\text {substituted) }\end{array}$ & $\begin{array}{l}\text { 4:-Ph } \\
\text { 5:-BPP }\end{array}$ & $\begin{array}{l}\mathrm{Pd}\left(\mathrm{PPh}_{3}\right)_{4} \\
\mathrm{Na}^{+} \mathrm{Ph}_{4} \mathrm{~B}^{-}\end{array}$ & anisole & $4 \cdot 3$ & 0.02 & 3.5 & 10 & 100 & - \\
\hline $\begin{array}{l}\text { norbornene } \\
\left(4,5^{-}\right. \\
\text {substituted) }\end{array}$ & $\begin{array}{l}\text { 4:-Ph } \\
\text { 5:-BPP }\end{array}$ & $\begin{array}{l}\mathrm{Pd}\left(\mathrm{PPh}_{3}\right)_{4} \\
\mathrm{Na}^{+} \mathrm{Ph}_{4} \mathrm{~B}^{-}\end{array}$ & anisole & 6.0 & 0.05 & 5.0 & 12 & 100 & - \\
\hline $\begin{array}{l}\text { norbornene } \\
\left(4,5^{-}\right. \\
\text {substituted) }\end{array}$ & $\begin{array}{l}\text { 4:-Ph } \\
\text { 5:-BPP }\end{array}$ & $\begin{array}{l}\mathrm{Pd}\left(\mathrm{PPh}_{3}\right)_{4} \\
\mathrm{Na}^{+} \mathrm{Ph}_{4} \mathrm{~B}^{-}\end{array}$ & anisole & 12.0 & 0.1 & 10.0 & 16 & 100 & - \\
\hline $\begin{array}{l}\text { norbornene } \\
\left(4,5^{-}\right. \\
\text {substituted })\end{array}$ & $\begin{array}{l}\text { 4:-Ph } \\
5:-P h\end{array}$ & $\begin{array}{l}\mathrm{Pd}(\mathrm{OAc})_{2} \\
\text { TEMPO }\end{array}$ & $\mathrm{AcOH}$ & 2.0 & 0.05 & 1.5 & 12 & 30 & - \\
\hline $\begin{array}{l}\text { norbornene } \\
\left(4,5^{-}\right. \\
\text {substituted })\end{array}$ & $\begin{array}{l}\text { 4:-Ph } \\
5:-\mathrm{Ph}\end{array}$ & $\begin{array}{l}\mathrm{Pd}(\mathrm{OAc})_{2} \\
\text { TEMPO }\end{array}$ & $\mathrm{AcOH}$ & 48.0 & 0.4 & 40.0 & 12 & 30 & - \\
\hline $\begin{array}{l}\text { norbornene } \\
\text { (4-substituted) }\end{array}$ & -isatin & $\begin{array}{l}\mathrm{PdCl}_{2}\left(\mathrm{PCy}_{3}\right)_{2} \\
\mathrm{NaHCO}_{2}\end{array}$ & DMF & 8.0 & 0.2 & 5.0 & 6 & 100 & $\begin{array}{l}35^{*} \\
\text { (impure) }\end{array}$ \\
\hline $\begin{array}{l}\text { norbornene } \\
\text { (4-substituted) }\end{array}$ & -isatin & $\begin{array}{l}\mathrm{PdCl}_{2}\left(\mathrm{PCy}_{3}\right)_{2} \\
\mathrm{NaHCO}_{2}\end{array}$ & DMF & 16.0 & 0.2 & 5.0 & 6 & 90 & - \\
\hline $\begin{array}{l}\text { norbornene } \\
\text { (4-substituted) }\end{array}$ & -isatin & $\begin{array}{l}\mathrm{PdCl}_{2}\left(\mathrm{PCy}_{3}\right)_{2} \\
\mathrm{NaHCO}_{2}\end{array}$ & DMF & 16.0 & 0.4 & 5.0 & 6 & 90 & $19^{*}$ \\
\hline $\begin{array}{l}\text { norbornene } \\
\text { (4-substituted) }\end{array}$ & -isatin & $\begin{array}{l}\mathrm{PdCl}_{2}\left(\mathrm{PCy}_{3}\right)_{2} \\
\mathrm{NaHCO}_{2}\end{array}$ & DMF & 16.0 & 0.2 & 10.0 & 6 & 90 & - \\
\hline
\end{tabular}




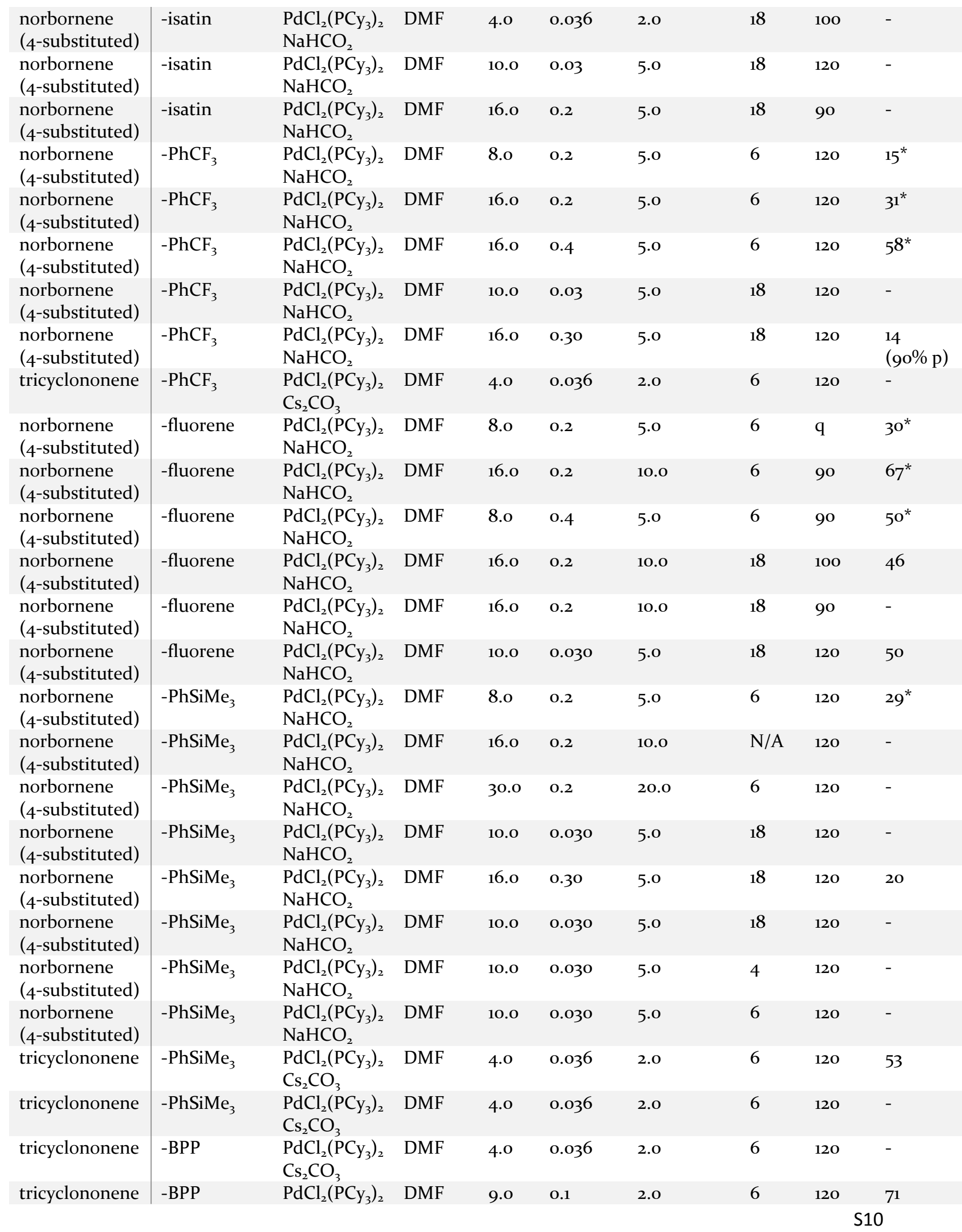




\begin{tabular}{|c|c|c|c|c|c|c|c|c|c|}
\hline & & $\mathrm{Cs}_{2} \mathrm{CO}_{3}$ & & & & & & & \\
\hline tricyclononene & -BPP & $\begin{array}{l}\mathrm{PdCl}_{2}\left(\mathrm{PCy}_{3}\right)_{2} \\
\mathrm{Cs}_{2} \mathrm{CO}_{3}\end{array}$ & DMF & 8.0 & 0.075 & 2.0 & 6 & 120 & 64 \\
\hline tricyclononene & -BPP & $\begin{array}{l}\mathrm{PdCl}_{2}\left(\mathrm{PCy}_{3}\right)_{2} \\
\mathrm{Cs}_{2} \mathrm{CO}_{3}\end{array}$ & DMF & 8.0 & 0.1 & 2.0 & 6 & 120 & 65 \\
\hline tricyclononene & -BPP & $\begin{array}{l}\mathrm{PdCl}_{2}\left(\mathrm{PCy}_{3}\right)_{2} \\
\mathrm{Cs}_{2} \mathrm{CO}_{3}\end{array}$ & DMF & 9.0 & 0.075 & 2.0 & 6 & 120 & 66 \\
\hline tricyclononene & -BPP & $\begin{array}{l}\mathrm{PdCl}_{2}\left(\mathrm{PCy}_{3}\right)_{2} \\
\mathrm{Cs}_{2} \mathrm{CO}_{3}\end{array}$ & DMF & 10.0 & 0.1 & 2.0 & 6 & 120 & 65 \\
\hline tricyclononene & $-B P P$ & $\begin{array}{l}\mathrm{PdCl}_{2}\left(\mathrm{PCy}_{3}\right)_{2} \\
\mathrm{Cs}_{2} \mathrm{CO}_{3}\end{array}$ & DMF & 4.0 & 0.036 & 2.0 & 20 & 120 & - \\
\hline tricyclononene & -BPP & $\begin{array}{l}\mathrm{PdCl}_{2}\left(\mathrm{PCy}_{3}\right)_{2} \\
\mathrm{Cs}_{2} \mathrm{CO}_{3}\end{array}$ & DMF & 4.0 & 0.036 & 2.0 & 6 & 120 & - \\
\hline tricyclononene & -BPP & $\begin{array}{l}\mathrm{PdCl}_{2}\left(\mathrm{PCy}_{3}\right)_{2} \\
\mathrm{Cs}_{2} \mathrm{CO}_{3}\end{array}$ & DMF & 6.0 & 0.036 & 2.0 & 6 & 120 & - \\
\hline tricyclononene & -ВРP & $\begin{array}{l}\mathrm{PdCl}_{2}\left(\mathrm{PCy}_{3}\right)_{2} \\
\mathrm{Cs}_{2} \mathrm{CO}_{3}\end{array}$ & DMF & 8.0 & 0.036 & 2.0 & 6 & 120 & - \\
\hline tricyclononene & -BPP & $\begin{array}{l}\mathrm{PdCl}_{2}\left(\mathrm{PCy}_{3}\right)_{2} \\
\mathrm{Cs}_{2} \mathrm{CO}_{3}\end{array}$ & DMF & 8.0 & 0.075 & 2.0 & 6 & 120 & - \\
\hline norbornene & -ВРP & $\begin{array}{l}\mathrm{PdCl}_{2}\left(\mathrm{PCy}_{3}\right)_{2} \\
\mathrm{NaHCO}_{2}\end{array}$ & DMF & 5.0 & 0.1 & 5.0 & 6 & 120 & $\mathrm{~N} / \mathrm{A}$ \\
\hline tricyclononene & -BPTMS & $\begin{array}{l}\mathrm{PdCl}_{2}\left(\mathrm{PCy}_{3}\right)_{2} \\
\mathrm{Cs}_{2} \mathrm{CO}_{3}\end{array}$ & DMF & 4.0 & 0.036 & 2.0 & 6 & 120 & 19 \\
\hline tricyclononene & -BPTMS & $\begin{array}{l}\mathrm{PdCl}_{2}\left(\mathrm{PCy}_{3}\right)_{2} \\
\mathrm{Cs}_{2} \mathrm{CO}_{3}\end{array}$ & DMF & 4.0 & 0.036 & 2.0 & 6 & 120 & 18 \\
\hline
\end{tabular}

Table S2. Polymer syntheses as a function of monomeric starting material and reaction conditions

\begin{tabular}{|c|c|c|c|c|c|c|c|c|c|}
\hline polymer & monomer & catalyst system & solvent & $\begin{array}{c}\text { Tim } \\
\text { e } \\
(\mathbf{h r})\end{array}$ & $\begin{array}{c}\text { Tem } \\
\mathbf{p} \\
\left({ }^{\circ} \mathrm{C}\right)\end{array}$ & $\begin{array}{c}\text { Yield } \\
(\%)\end{array}$ & $\mathbf{M}_{\mathrm{W}}$ & $M_{n}$ & $\mathbf{M}_{\mathbf{w}} / \mathbf{M}_{\mathbf{n}}$ \\
\hline $\begin{array}{l}\text { APN- } \\
\text { BPP }\end{array}$ & $\begin{array}{l}\text { propoxy } \\
\text { norbornene }\end{array}$ & $\begin{array}{l}\mathrm{Cp}_{\mathrm{p}}(\mathrm{Pd}) \text { phenylall } \\
\text { yl } \\
\text { Trityl BArF/PCy }\end{array}$ & toluene & 24 & 100 & 80 & & not measured & \\
\hline $\begin{array}{l}\text { APN- } \\
\text { BPP }\end{array}$ & $\begin{array}{l}\text { propoxy } \\
\text { norbornene }\end{array}$ & $\begin{array}{l}\mathrm{Cp}(\mathrm{Pd}) \text { phenylall } \\
\text { yl } \\
\text { Trityl BArF/ } / \mathrm{PCy}_{3}\end{array}$ & toluene & 24 & 100 & 44 & $7.20 \times 10^{5}$ & $2.68 \times 10^{5}$ & 2.69 \\
\hline $\begin{array}{l}\text { APN- } \\
\text { BPP }\end{array}$ & $\begin{array}{l}\text { propoxy } \\
\text { norbornene }\end{array}$ & $\begin{array}{l}\mathrm{Cp}(\mathrm{Pd}) \text { phenylall } \\
\text { yl } \\
\text { Trityl BArF/PCy }\end{array}$ & toluene & 24 & 100 & 88 & & not measured & \\
\hline $\begin{array}{l}\text { APTCN- } \\
\text { BPP }\end{array}$ & $\begin{array}{l}\text { propoxy } \\
\text { tricyclononen } \\
\text { e }\end{array}$ & $\begin{array}{l}\mathrm{Cp}(\mathrm{Pd}) \text { phenylall } \\
\text { yl } \\
\text { Trityl BArF/PCy }\end{array}$ & toluene & 24 & 100 & 80 & & not measured & \\
\hline $\begin{array}{l}\text { APTCN- } \\
\text { BPP }\end{array}$ & $\begin{array}{l}\text { propoxy } \\
\text { tricyclononen } \\
\text { e }\end{array}$ & $\begin{array}{l}\mathrm{Cp}(\mathrm{Pd}) \text { phenylall } \\
\text { yl } \\
\text { Trityl BArF/PCy }\end{array}$ & toluene & 18 & 100 & $\begin{array}{l}>99 \\
\%\end{array}$ & $1.78 \times 10^{6}$ & $1.08 \times 10^{6}$ & 1.66 \\
\hline $\begin{array}{l}\text { APTCN- } \\
\text { BPTMS }\end{array}$ & $\begin{array}{l}\text { TMS } \\
\text { tricyclononen } \\
\mathrm{e}\end{array}$ & $\begin{array}{l}\mathrm{Cp}(\mathrm{Pd}) \text { phenylall } \\
\text { yl } \\
\text { Trityl BArF } / \mathrm{PCy}_{3}\end{array}$ & toluene & 18 & 100 & $\begin{array}{l}\text { No } \\
\text { RXN }\end{array}$ & & not measured & \\
\hline
\end{tabular}




\begin{tabular}{|c|c|c|c|c|c|c|c|c|c|}
\hline $\begin{array}{l}\text { APTCN- } \\
\text { BPTMS }\end{array}$ & $\begin{array}{l}\text { TMS } \\
\text { tricyclononen } \\
\text { e }\end{array}$ & $\begin{array}{l}\mathrm{Cp}(\mathrm{Pd}) \text { phenylall } \\
\text { yl } \\
\text { Trityl BArF/PCy }\end{array}$ & toluene & 18 & 100 & 67 & $1.49 \times 10^{6}$ & $0.85 \times 10^{6}$ & 1.74 \\
\hline $\begin{array}{l}\text { ROMP- } \\
\text { N-BPP }\end{array}$ & $\begin{array}{l}\text { Propoxy } \\
\text { norbornene }\end{array}$ & Grubbs $3^{\text {rd }}$ gen. & toluene & 18 & 40 & 72 & & not measured & \\
\hline $\begin{array}{l}\text { ROMP- } \\
\text { N-BPP }\end{array}$ & $\begin{array}{l}\text { Propoxy } \\
\text { norbornene }\end{array}$ & Grubbs $1^{\text {st }}$ gen. & toluene & 18 & 40 & 88 & $2.45 \times 10^{5}$ & $0.64 X_{10}^{5}$ & 3.83 \\
\hline $\begin{array}{l}\text { ROMP- } \\
\text { TCN- } \\
\text { BPP }\end{array}$ & $\begin{array}{l}\text { propoxy } \\
\text { tricyclononen } \\
\text { e }\end{array}$ & Grubbs $1^{\text {st }}$ gen. & toluene & 18 & 40 & 72 & & not measured & \\
\hline $\begin{array}{l}\text { ROMP- } \\
\text { TCN- } \\
\text { BPP }\end{array}$ & $\begin{array}{l}\text { Propoxy } \\
\text { tricyclononen } \\
\text { e }\end{array}$ & Grubbs $1^{\text {st }}$ gen. & toluene & 18 & 40 & 94 & $3.53 \times 10^{5}$ & $0.90 \times 10^{5}$ & 3.93 \\
\hline
\end{tabular}

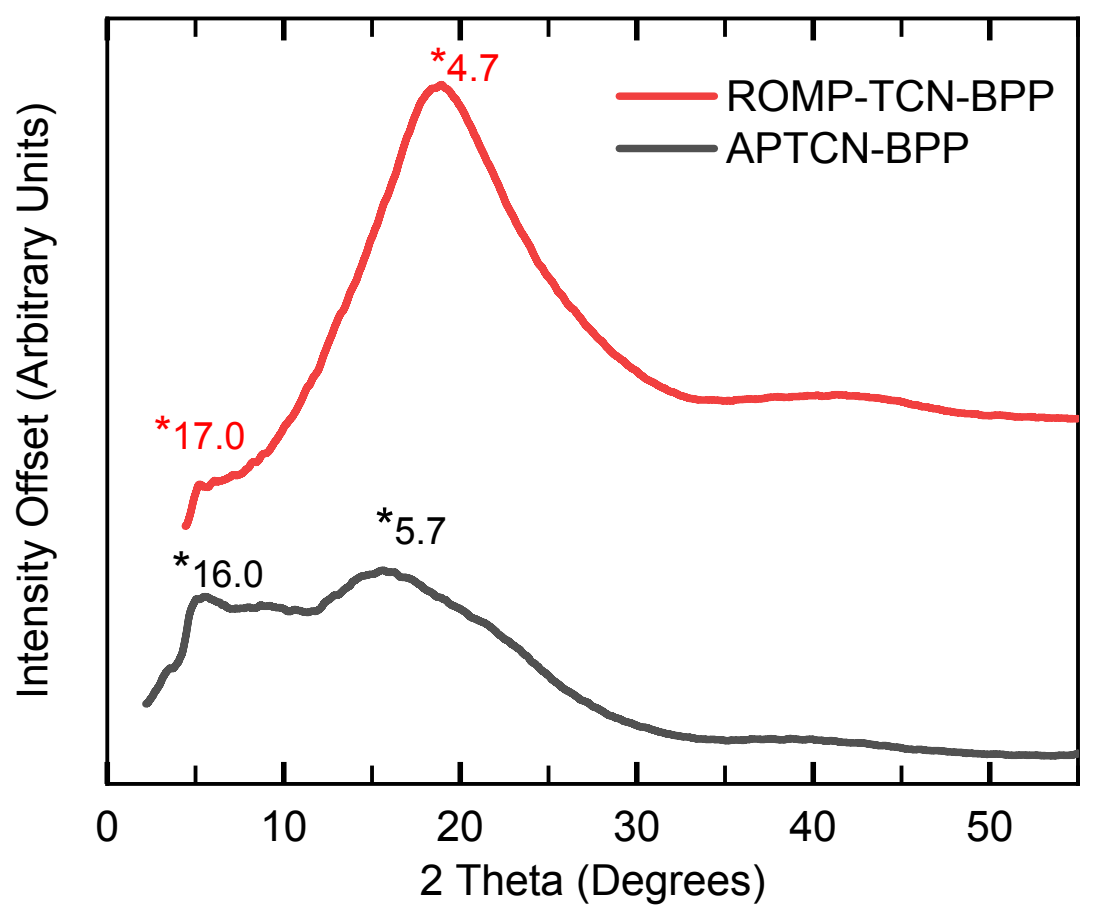

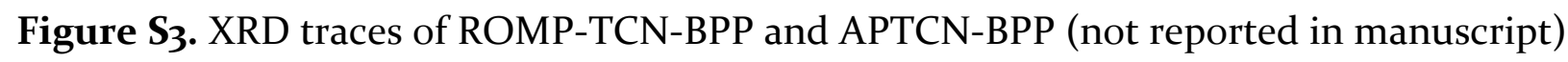




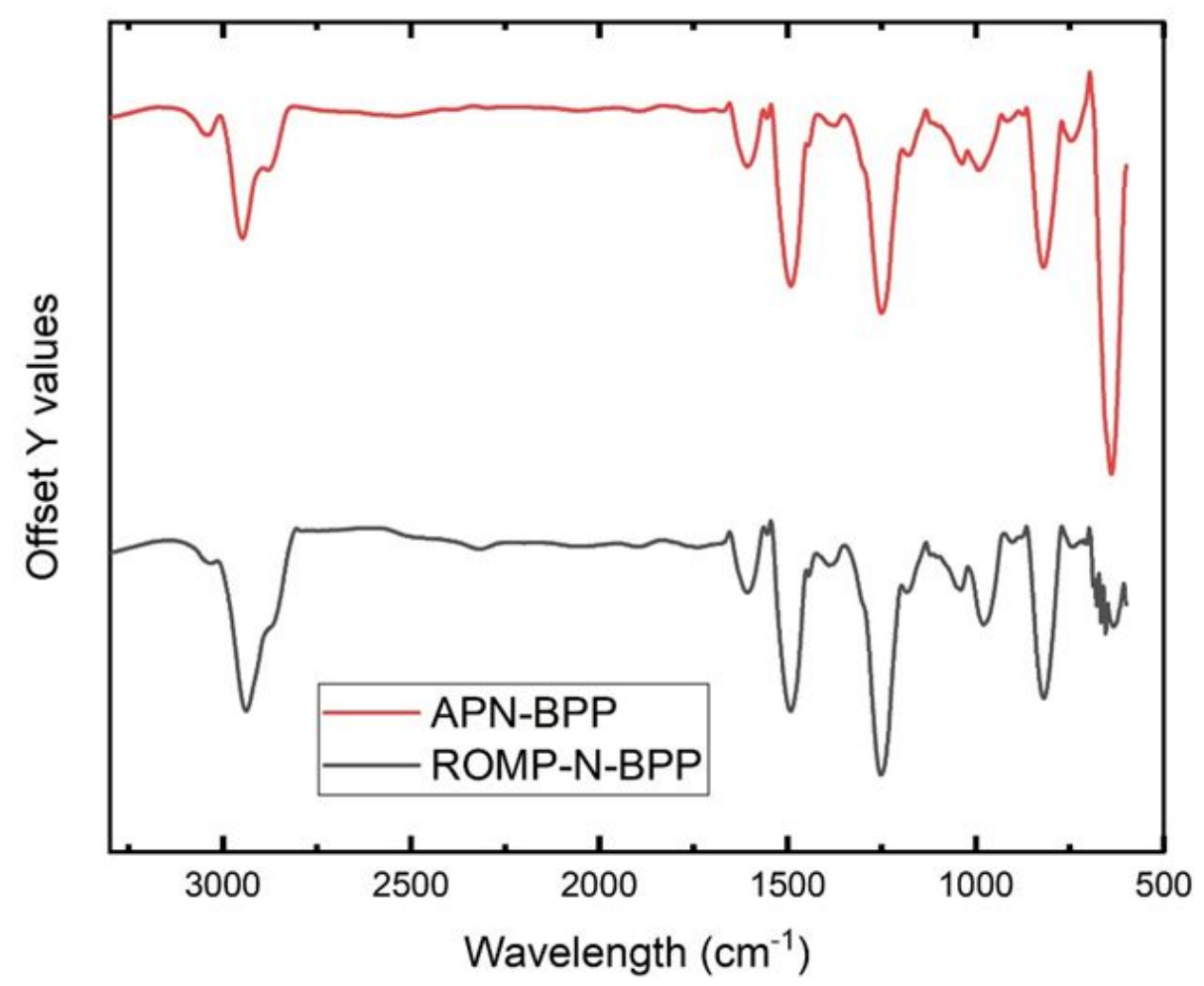

Figure S4. FTIR spectra comparing addition-type and ROMP polymers using biphenyl propoxy norbornene monomers

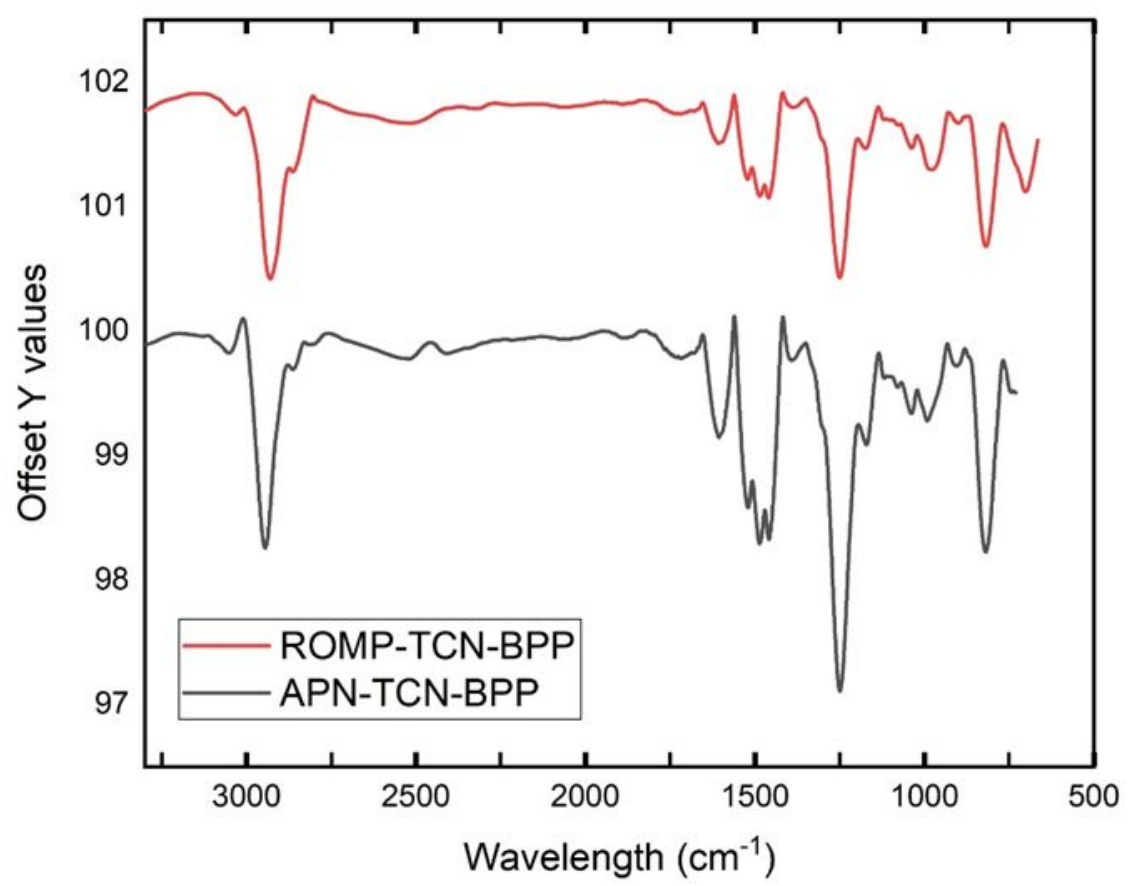

Figure S5. FTIR spectra comparing addition-type and ROMP polymers using biphenyl propoxy tricyclononene monomers 


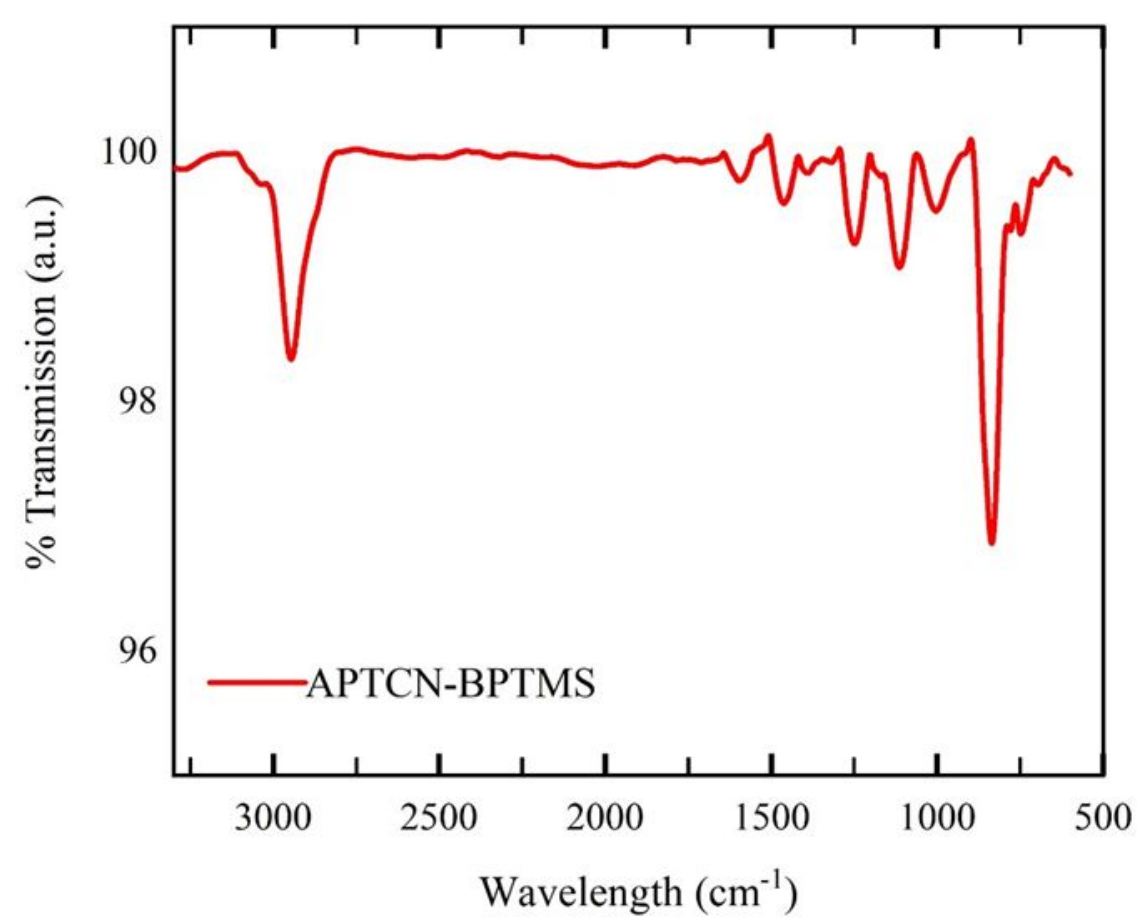

Figure S6. FTIR spectra of the addition-type polymers using trimethylsilyl substituted tricyclononene monomer

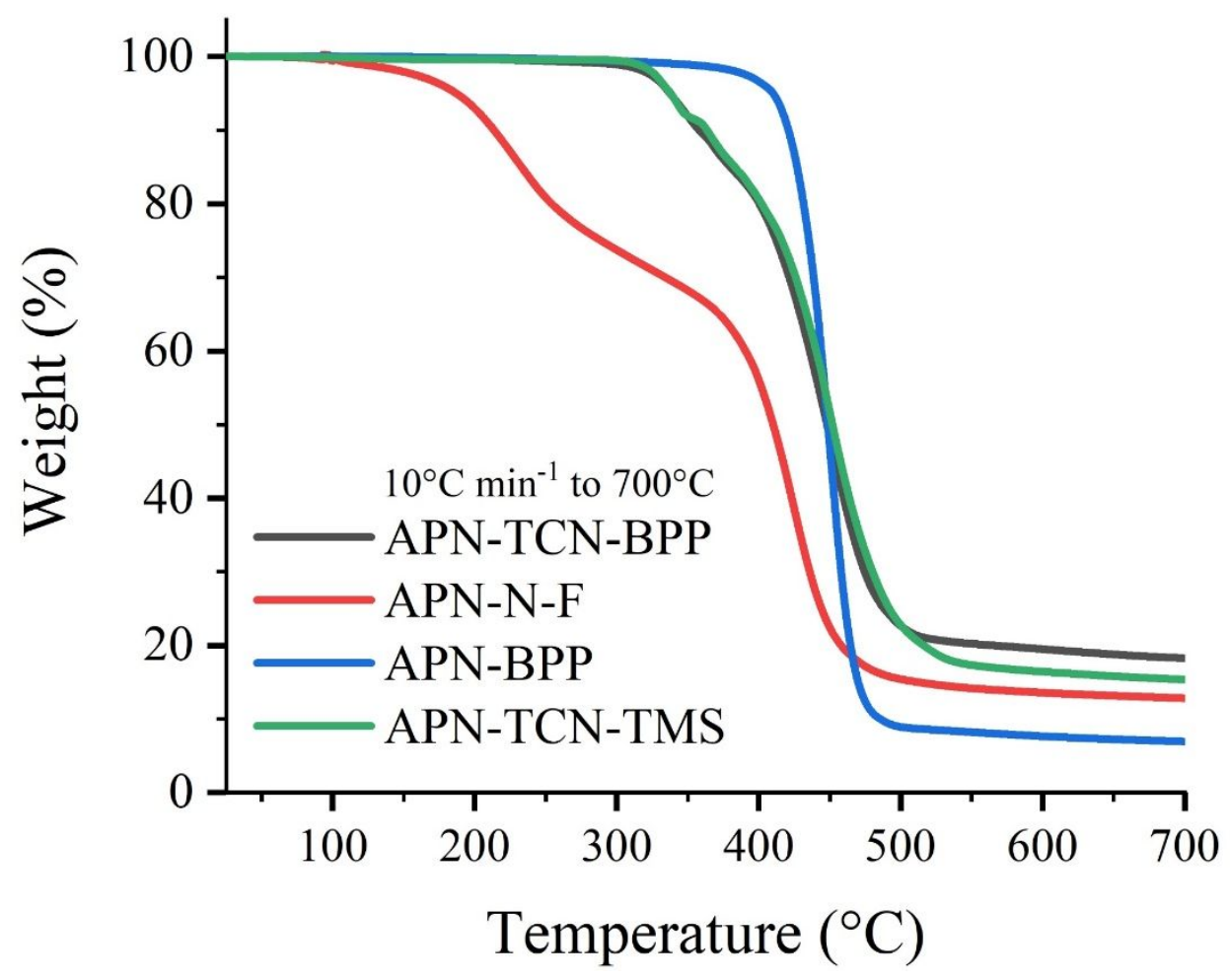

Figure $S_{7}$. TGA thermograms comparing the addition-type polymers showed consistently high thermal stabilities (excluding APN-N-F, and impure fluorene based polynorbornene) 


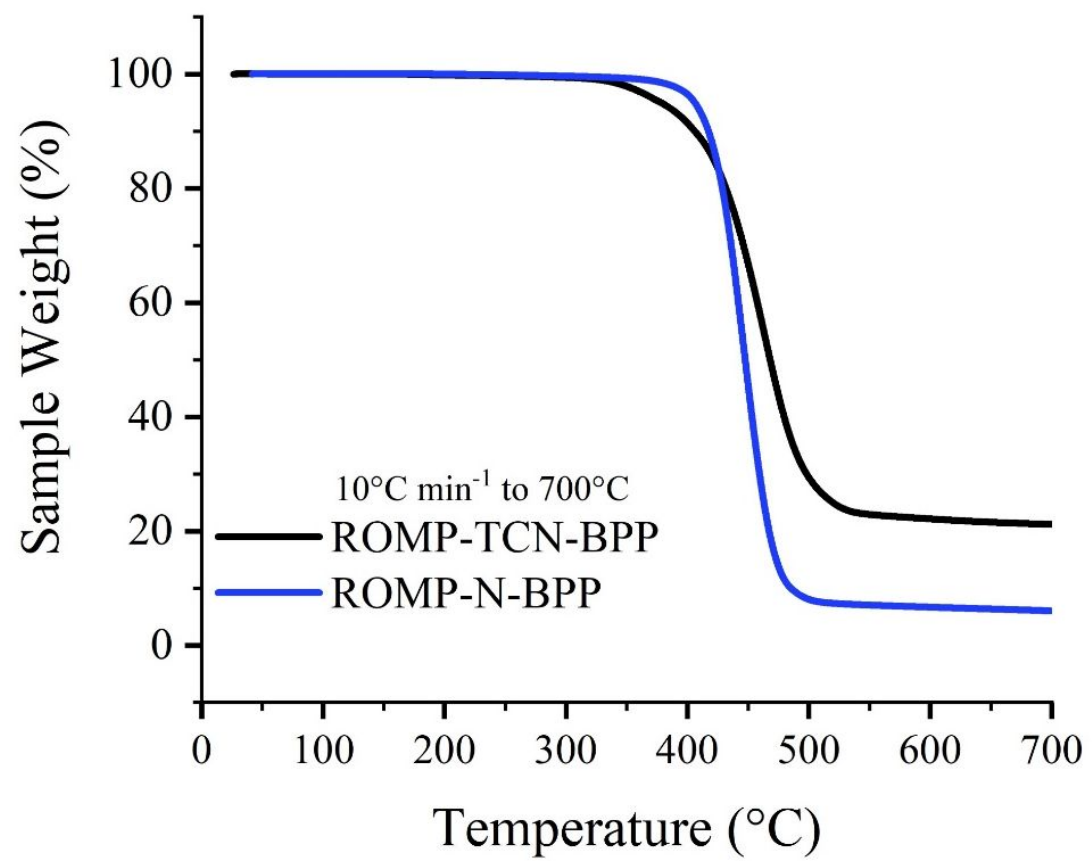

Figure S8. TGA thermograms comparing the ROMP polymers showed consistently high thermal stabilities

Table S3: Thermogravimetric Analysis (TGA) Degradation Temperatures and Residual Masses

Thermogravimetric analysis

\begin{tabular}{lccc}
\cline { 2 - 4 } \multicolumn{1}{c}{ Polymer } & $\begin{array}{c}5 \% \text { Weight Loss } \\
\left({ }^{\circ} \mathrm{C}\right)\end{array}$ & $\begin{array}{c}10 \% \text { Weight Loss } \\
\left({ }^{\circ} \mathrm{C}\right)\end{array}$ & $\begin{array}{c}\text { Residual Mass } \\
(\%)\end{array}$ \\
\hline APN-TCN-BPP & $338^{\circ} \mathrm{C}$ & $358{ }^{\circ} \mathrm{C}$ & $18.3 \%$ \\
APN-N-BPP & $410^{\circ} \mathrm{C}$ & $421{ }^{\circ} \mathrm{C}$ & $6.9 \%$ \\
APN-N-F & $187{ }^{\circ} \mathrm{C}$ & $213{ }^{\circ} \mathrm{C}$ & $12.8 \%$ \\
APN-TCN-BPTMS & $338^{\circ} \mathrm{C}$ & $364^{\circ} \mathrm{C}$ & $15.4 \%$ \\
ROMP-TCN-BPP & $378^{\circ} \mathrm{C}$ & $406{ }^{\circ} \mathrm{C}$ & $21.2 \%$ \\
ROMP-N-BPP & $406{ }^{\circ} \mathrm{C}$ & $417{ }^{\circ} \mathrm{C}$ & $6.1 \%$ \\
\hline
\end{tabular}




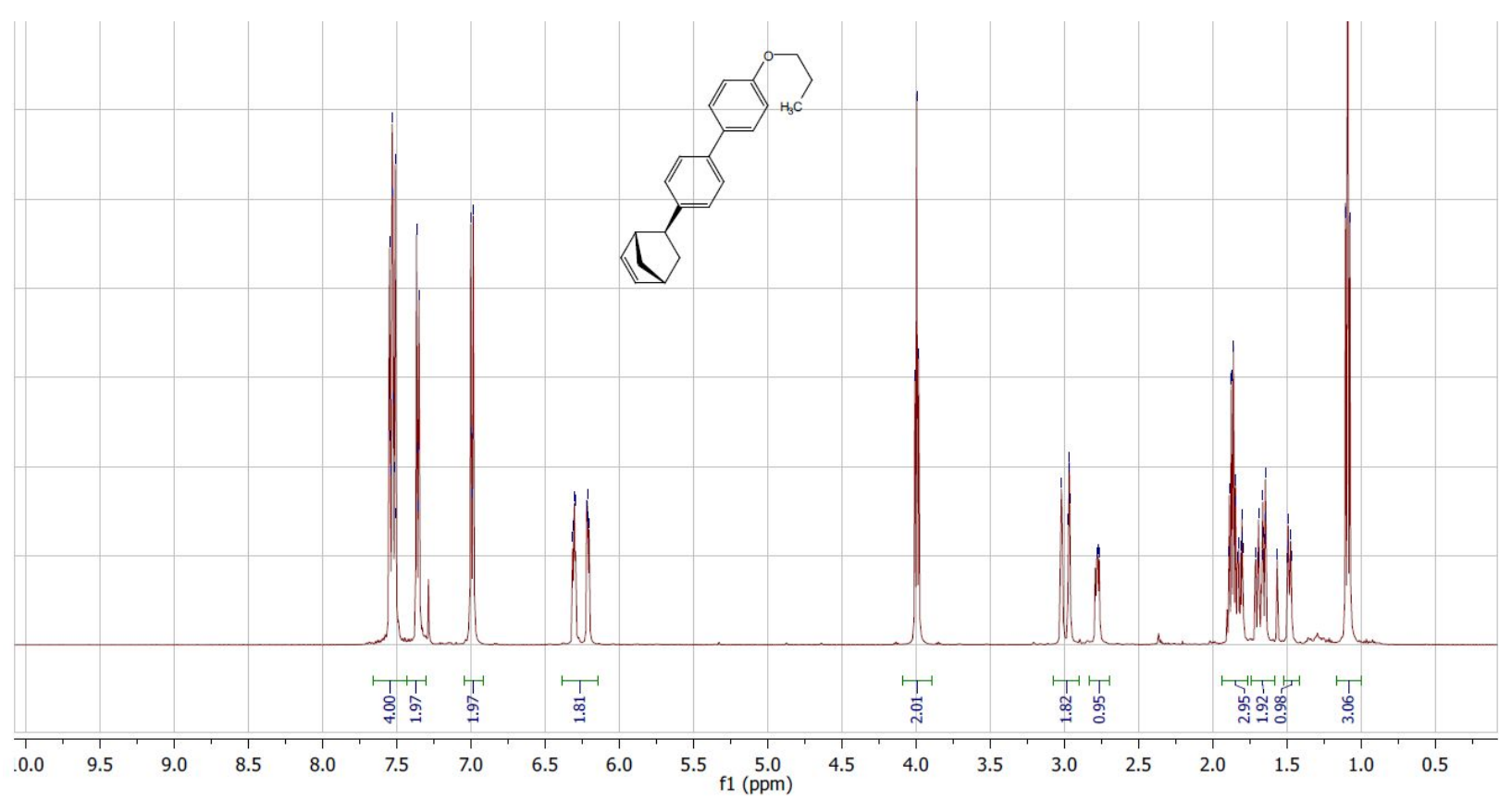

Figure S9. ${ }^{1} \mathrm{H}$-NMR spectra of 4-biphenylpropoxy norbornene ${ }^{1} \mathrm{H}$ NMR $\left(\mathrm{CDCl}_{3}, 296 \mathrm{~K}\right): \delta(\mathrm{ppm})-7.41(4 \mathrm{H}, \mathrm{m}), 7.25(2 \mathrm{H}, \mathrm{m}), 6.88(2 \mathrm{H}, \mathrm{m}), 6.18\left({ }_{1} \mathrm{H}, \mathrm{m}\right)$, $6.10(1 \mathrm{H}, \mathrm{m}), 3.88(2 \mathrm{H}, \mathrm{t}), 2.90(1 \mathrm{H}, \mathrm{s}), 2.85(1 \mathrm{H}, \mathrm{s}), 2.67(1 \mathrm{H}, \mathrm{m}), 1.75(2 \mathrm{H}, \mathrm{m}), 1.69(1 \mathrm{H}, \mathrm{m})$, $1.59(1 \mathrm{H}, \mathrm{m}), 1.54(\mathrm{iH}, \mathrm{m}), 1.37(\mathrm{HH}, \mathrm{m}), 0.98(3 \mathrm{H}, \mathrm{t})$

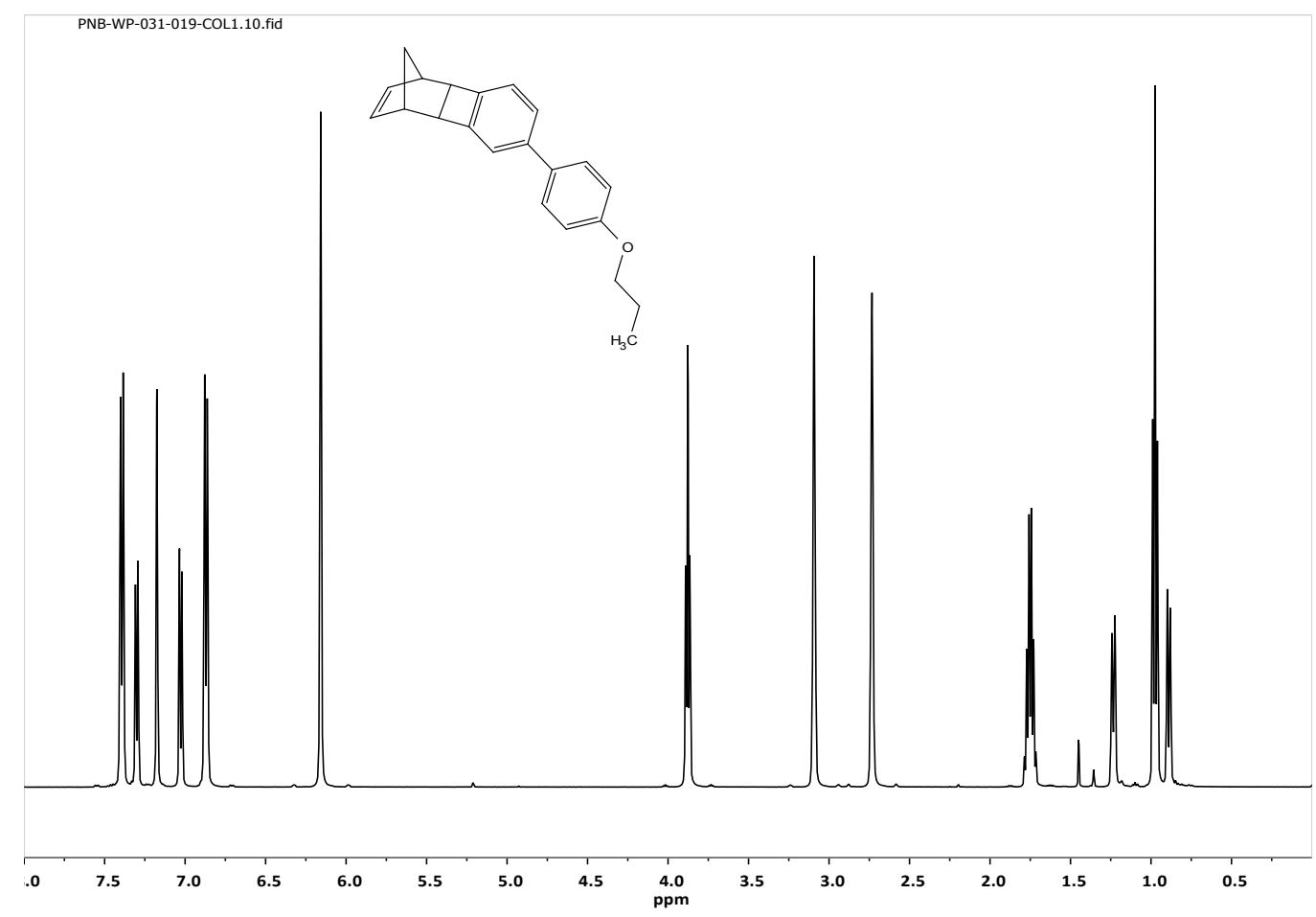

Figure S1o. ${ }^{1} \mathrm{H}-\mathrm{NMR}$ spectra of 4-biphenylpropoxy tricyclononene 
${ }^{1} \mathrm{H}$ NMR $\left(\mathrm{CDCl}_{3}, 296 \mathrm{~K}\right): \delta(\mathrm{ppm})-7.39(2 \mathrm{H}, \mathrm{m}), 7.30(1 \mathrm{H}, \mathrm{m}), 7.18(\mathrm{HH}, \mathrm{s}), 7.03(1 \mathrm{H}, \mathrm{m})$, $6.87(2 \mathrm{H}, \mathrm{m}), 6.16(2 \mathrm{H}, \mathrm{s}), 3.88(2 \mathrm{H}, \mathrm{t}), 3.09(2 \mathrm{H}, \mathrm{s}), 2.73(2 \mathrm{H}, \mathrm{s}), 1.75(2 \mathrm{H}, \mathrm{m}), 1.23(1 \mathrm{H}, \mathrm{d})$, $0.97(3 \mathrm{H}, \mathrm{t}), 0.89(\mathrm{iH}, \mathrm{d})$

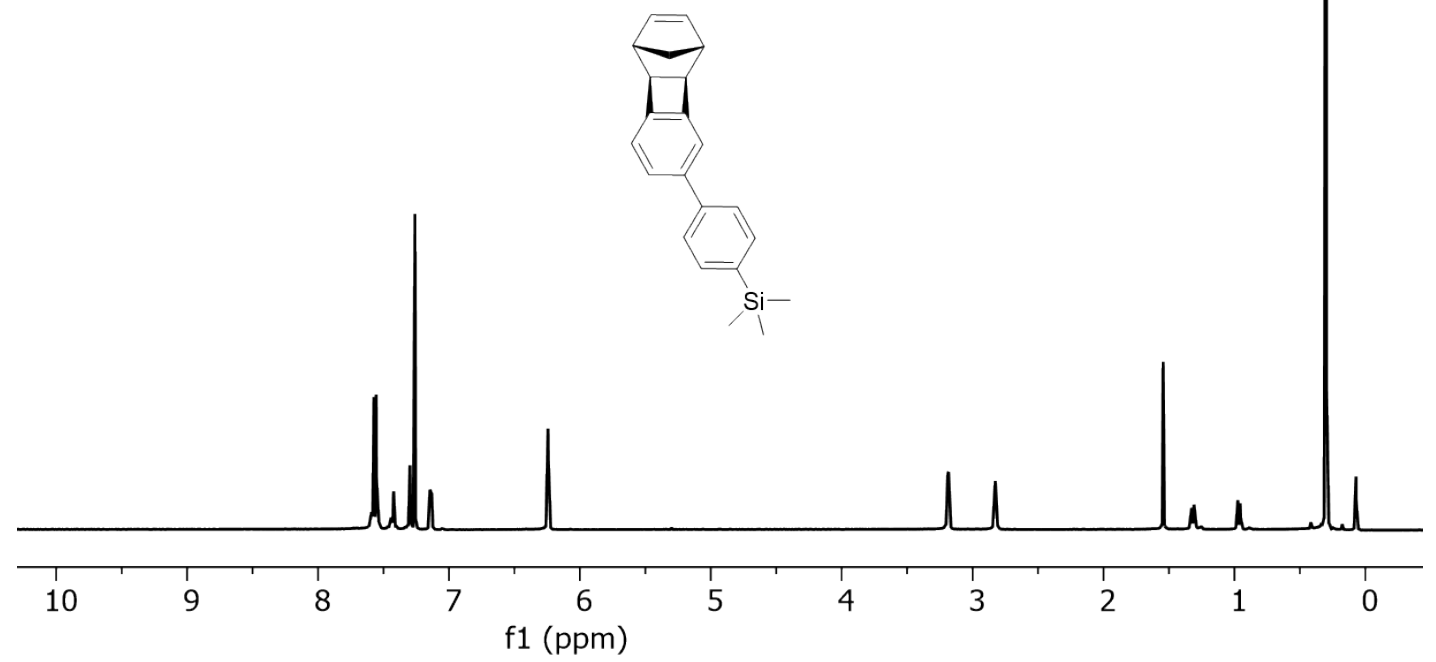

Figure S11. ${ }^{1} \mathrm{H}-\mathrm{NMR}$ spectra of 4-biphenyltrimethylsilyl tricyclononene ${ }^{1} \mathrm{H}$ NMR $\left(\mathrm{CDCl}_{3}, 296 \mathrm{~K}\right): \delta(\mathrm{ppm})-7.57(4 \mathrm{H}, \mathrm{m}), 7.43(1 \mathrm{H}, \mathrm{m}), 7.30(1 \mathrm{H}, \mathrm{s}), 7.14(1 \mathrm{H}, \mathrm{m})$, $6.24(2 \mathrm{H}, \mathrm{m}), 3.19(2 \mathrm{H}, \mathrm{m}), 2.82(2 \mathrm{H}, \mathrm{m}), 1.32(\mathrm{H}, \mathrm{m}), 0.96(1 \mathrm{H}, \mathrm{m}), 0.30(9 \mathrm{H}, \mathrm{s})$

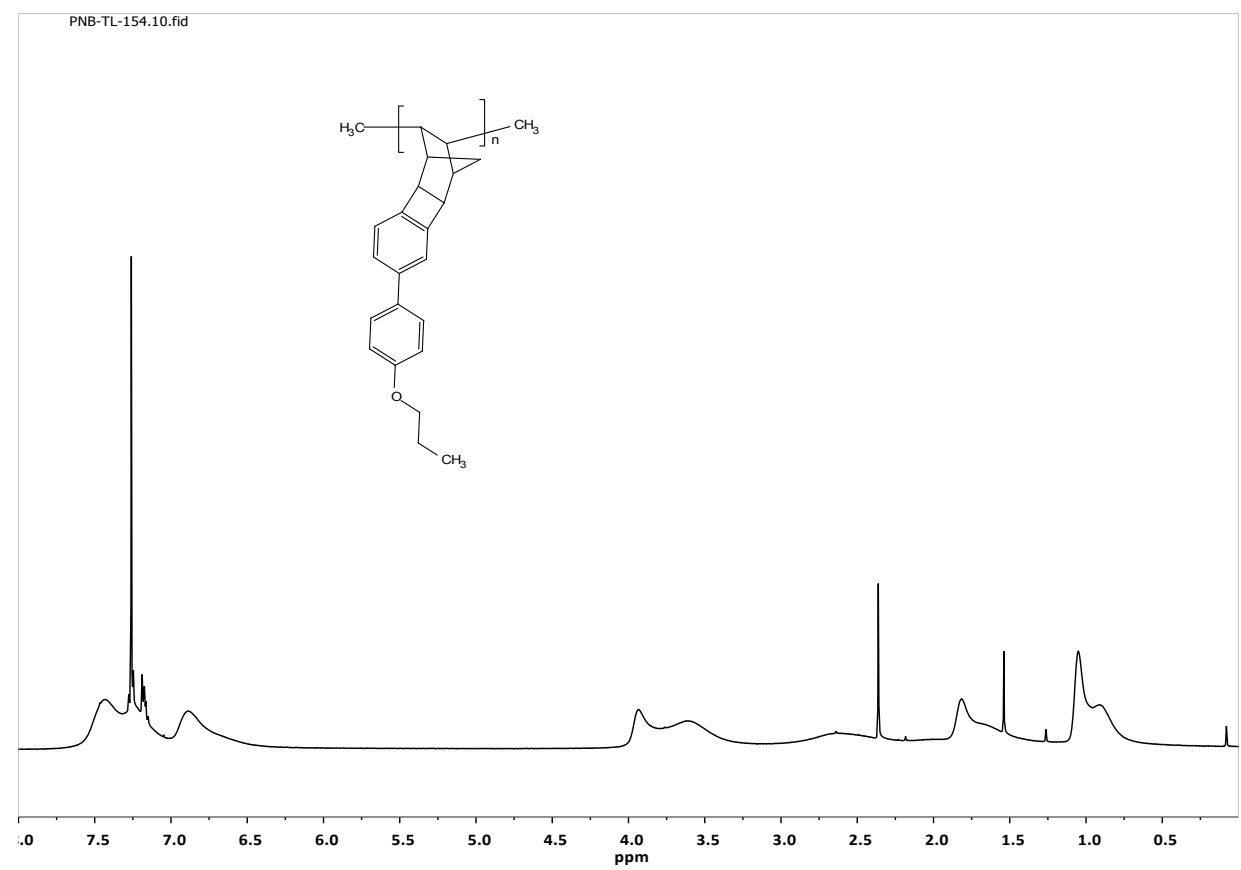

Figure S12. ${ }^{1} \mathrm{H}-\mathrm{NMR}$ spectra of APTCN-BPP 
${ }^{1} \mathrm{H} \mathrm{NMR}\left(\mathrm{CDCl}_{3}, 296 \mathrm{~K}\right): \delta(\mathrm{ppm})-7.35(5 \mathrm{H}, \mathrm{br}), 6.81(2 \mathrm{H}, \mathrm{br}), 3.85(2 \mathrm{H}, \mathrm{br}), 3.53(3 \mathrm{H}, \mathrm{br})$, $2.56(2 \mathrm{H}, \mathrm{br}), 1.74(2 \mathrm{H}, \mathrm{br}), 1.59(1 \mathrm{H}, \mathrm{br}), 0.97$ ( $3 \mathrm{H}, \mathrm{br}), 0.84(2 \mathrm{H}, \mathrm{br})$

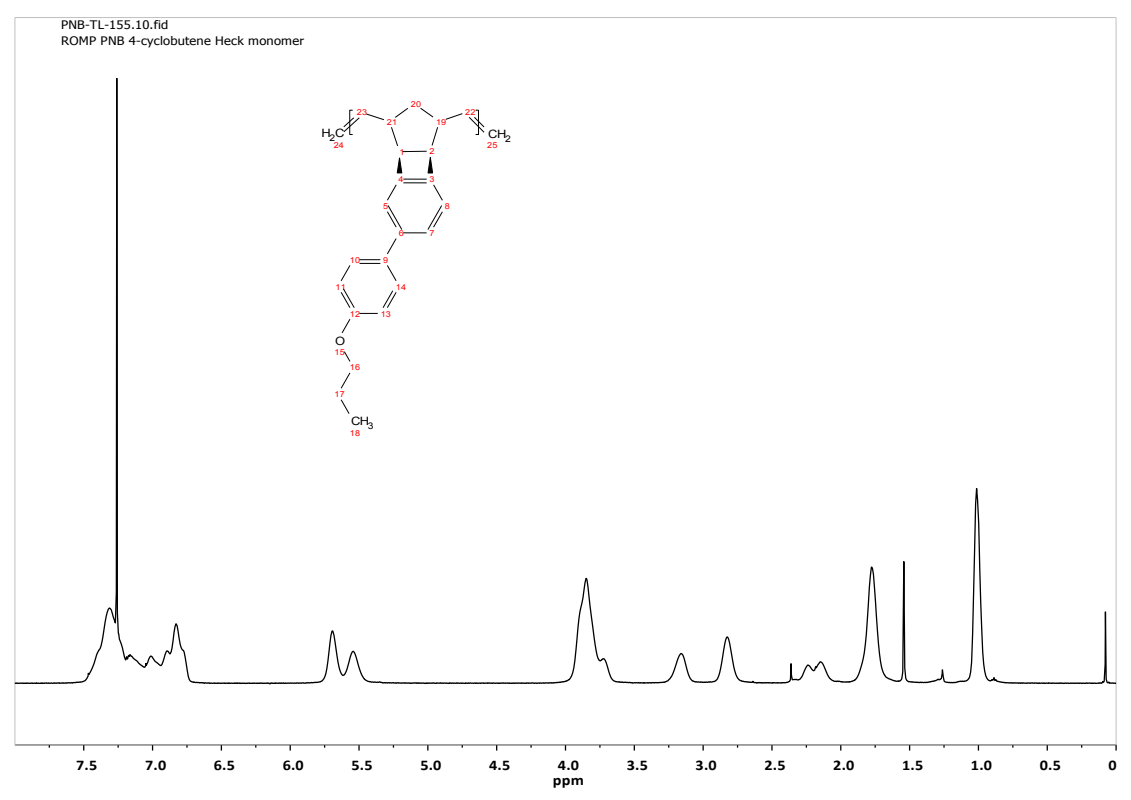

Figure S13. ${ }^{1} \mathrm{H}-\mathrm{NMR}$ spectra of ROMP-TCN-BPP

${ }^{1} \mathrm{H}$ NMR $\left(\mathrm{CDCl}_{3}, 296 \mathrm{~K}\right): \delta(\mathrm{ppm})$ - $7.24(4 \mathrm{H}, \mathrm{br}), 6.94(1 \mathrm{H}, \mathrm{br}), 6.75(2 \mathrm{H}, \mathrm{m}, \mathrm{br}), 5.62(1 \mathrm{H}$, br), $5.47(1 \mathrm{H}$, br), $3.78(2 \mathrm{H}, \mathrm{br}), 3.65(2 \mathrm{H}, \mathrm{br}), 3.08(1 \mathrm{H}, \mathrm{br}), 2.75(1 \mathrm{H}, \mathrm{br}), 2.11(1 \mathrm{H}, \mathrm{m}, \mathrm{br})$, $1.70(3 \mathrm{H}, \mathrm{br}), 0.94(3 \mathrm{H}, \mathrm{br})$

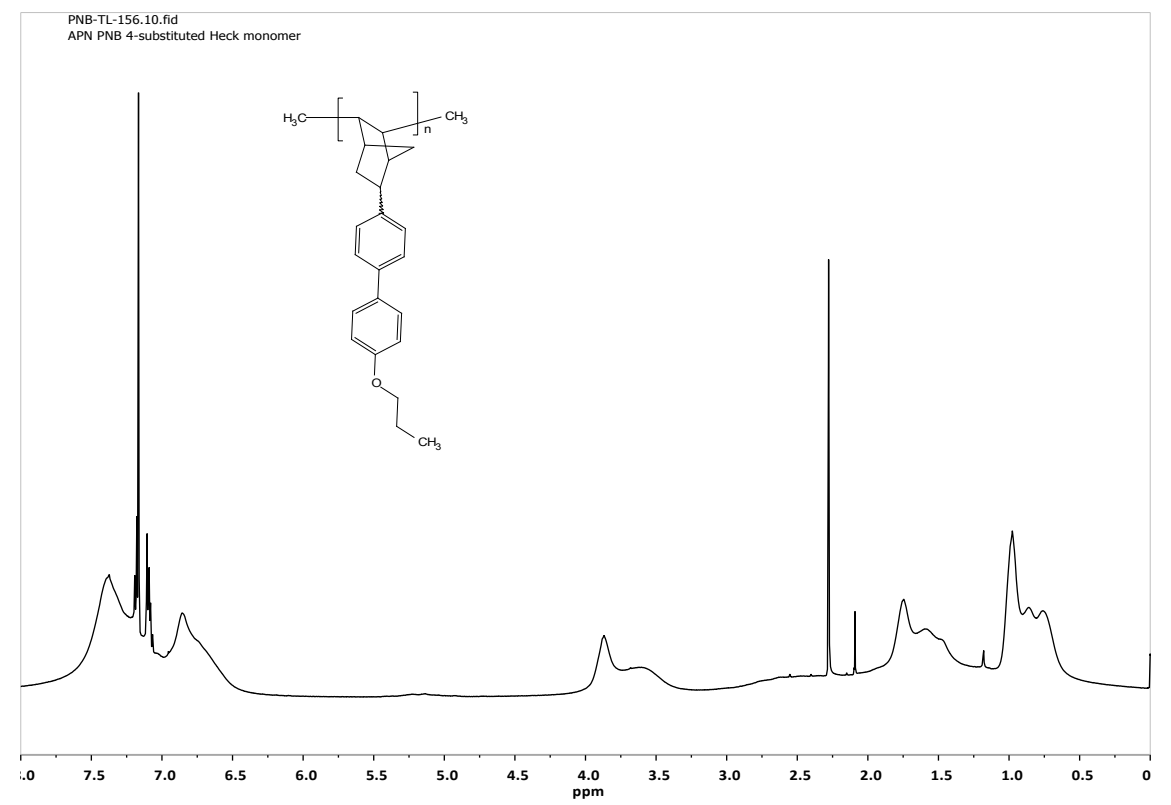

Figure S14. ${ }^{1} \mathrm{H}-\mathrm{NMR}$ spectra of APN-BPP 
${ }^{1} \mathrm{H} \mathrm{NMR}\left(\mathrm{CDCl}_{3}, 296 \mathrm{~K}\right): \delta(\mathrm{ppm})-7.38(5 \mathrm{H}, \mathrm{br}), 6.85(3 \mathrm{H}, \mathrm{br}), 3.88(2 \mathrm{H}, \mathrm{br}), 3.58$ (3H, br), $2.56(2 \mathrm{H}, \mathrm{br}), 1.75(2 \mathrm{H}, \mathrm{br}), 1.59(1 \mathrm{H}, \mathrm{br}), 0.98(3 \mathrm{H}, \mathrm{br}), 0.86(1 \mathrm{H}, \mathrm{br}), 0.77(2 \mathrm{H}, \mathrm{br})$

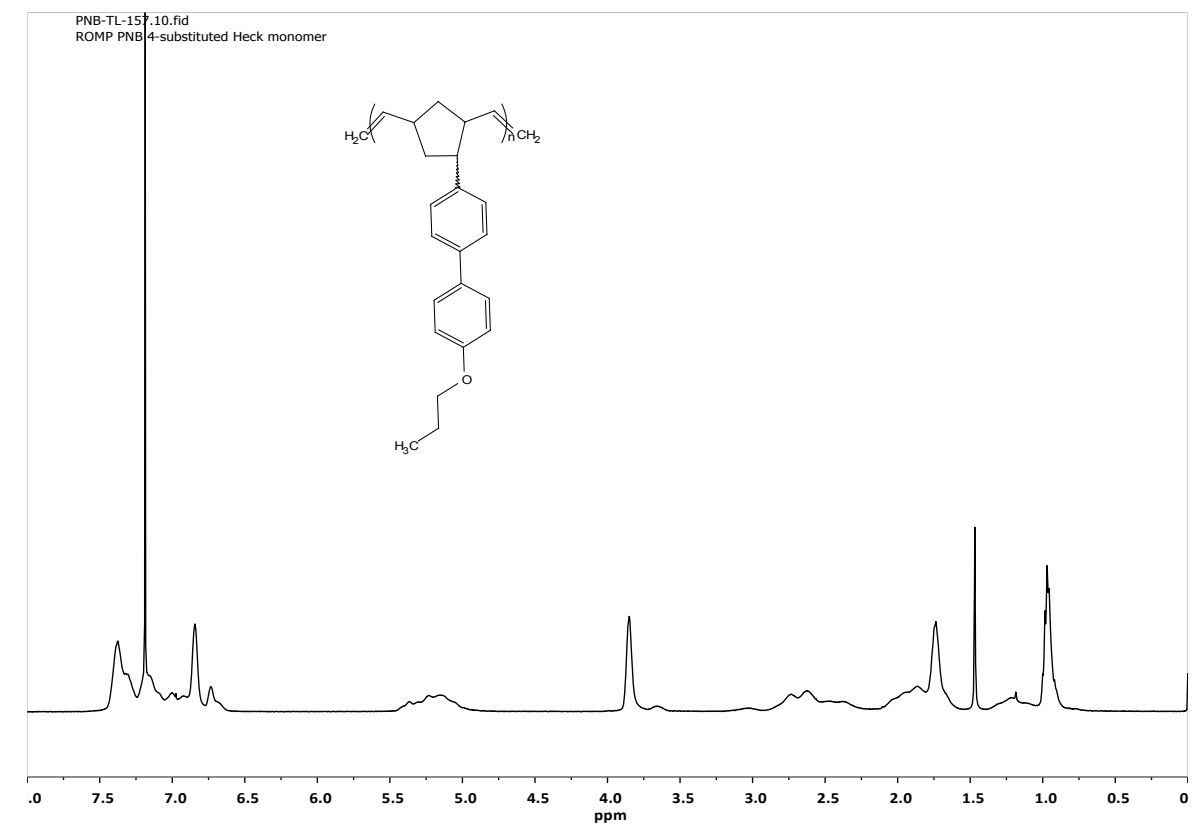

Figure S15. ${ }^{1} \mathrm{H}-\mathrm{NMR}$ spectra of ROMP-N-BPP

${ }^{1} \mathrm{H}$ NMR $\left(\mathrm{CDCl}_{3}, 296 \mathrm{~K}\right): \delta(\mathrm{ppm})-7.37(3 \mathrm{H}, \mathrm{br}), 7.15(2 \mathrm{H}, \mathrm{m}, \mathrm{br}), 6.84(2 \mathrm{H}, \mathrm{br}), 6.73(1 \mathrm{H}$, br), $5.23(2 \mathrm{H}, \mathrm{m}, \mathrm{br}), 3.85(2 \mathrm{H}, \mathrm{br}), 2.74(1 \mathrm{H}, \mathrm{br}), 2.62(1 \mathrm{H}, \mathrm{br}), 2.36(1 \mathrm{H}, \mathrm{br}), 1.99(1 \mathrm{H}, \mathrm{br})$, $1.87(\mathrm{H}, \mathrm{br}), 1.73(3 \mathrm{H}, \mathrm{br}), 1.22(\mathrm{H}, \mathrm{br}), 0.97(3 \mathrm{H}, \mathrm{br})$

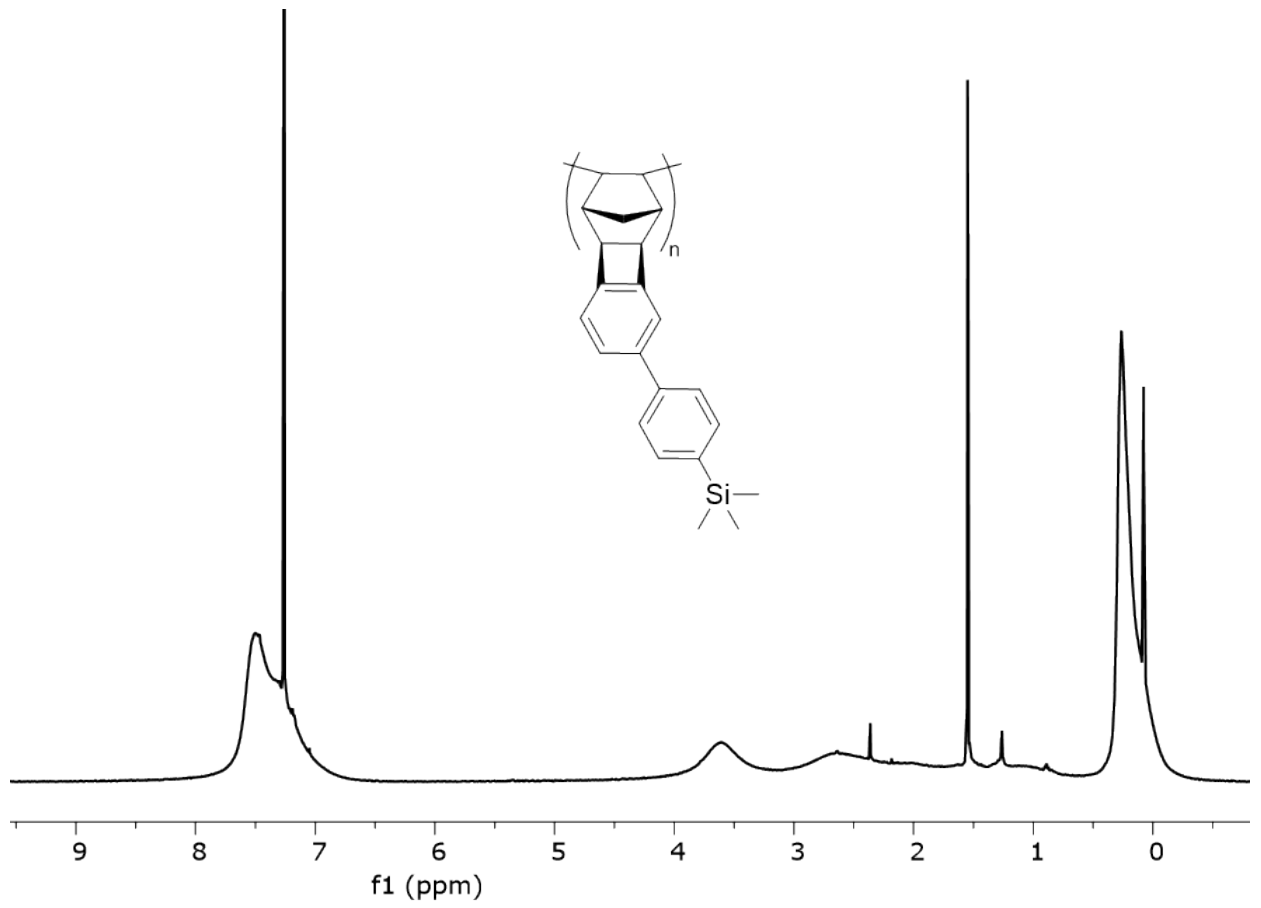

Figure S16. ${ }^{1} \mathrm{H}-\mathrm{NMR}$ spectra of APNTCN-BPTMS 
$\left.{ }^{1} \mathrm{H} \mathrm{NMR} \mathrm{(CDCl}, 296 \mathrm{~K}\right): \delta(\mathrm{ppm})-7.43(7 \mathrm{H}, \mathrm{br}), 3.54(2 \mathrm{H}, \mathrm{br}), 2.55(2 \mathrm{H}, \mathrm{br}), 2.00(2 \mathrm{H}, \mathrm{br})$, $1.04(2 \mathrm{H}, \mathrm{br}), 0.19(9 \mathrm{H}, \mathrm{br})$

Table S4: Elemental analysis results from the addition-type and ROMP polymers showed close matches between theoretical and experimental atomic composition

Elemental Analysis

\begin{tabular}{cccc}
\cline { 2 - 3 } Polymer & $\begin{array}{c}\text { Carbon } \% \\
\text { (theoretical, } \\
\text { experimental) }\end{array}$ & $\begin{array}{c}\text { Hydrogen \% (theoretical, } \\
\text { experimental) }\end{array}$ & $\begin{array}{c}\text { Oxygen \% (theoretical, } \\
\text { experimental) }\end{array}$ \\
\hline $\begin{array}{c}\text { ROMP-N- } \\
\text { BPP }\end{array}$ & $(86.80,85.93)$ & $(7.95,7.58)$ & $(5.26,5.34)$ \\
ROMP- & $(87.38,86.19)$ & $(7.33,7.41)$ & $(5.29,6.30)$ \\
TCN-BPP & $(7.95,7.46)$ & $(5.26,5.38)$ \\
$\begin{array}{c}\text { APN-BPP } \\
\text { APTCN- } \\
\text { BPP }\end{array}$ & $(86.80,85.56)$ & $(7.33,7.23)$ & $(5.29,5.08)$ \\
$\begin{array}{c}(8 P T C N- \\
\text { BPTMS }\end{array}$ & $(83.18,82.83)$ & $(7.64,7.58)$ & \\
\hline
\end{tabular}

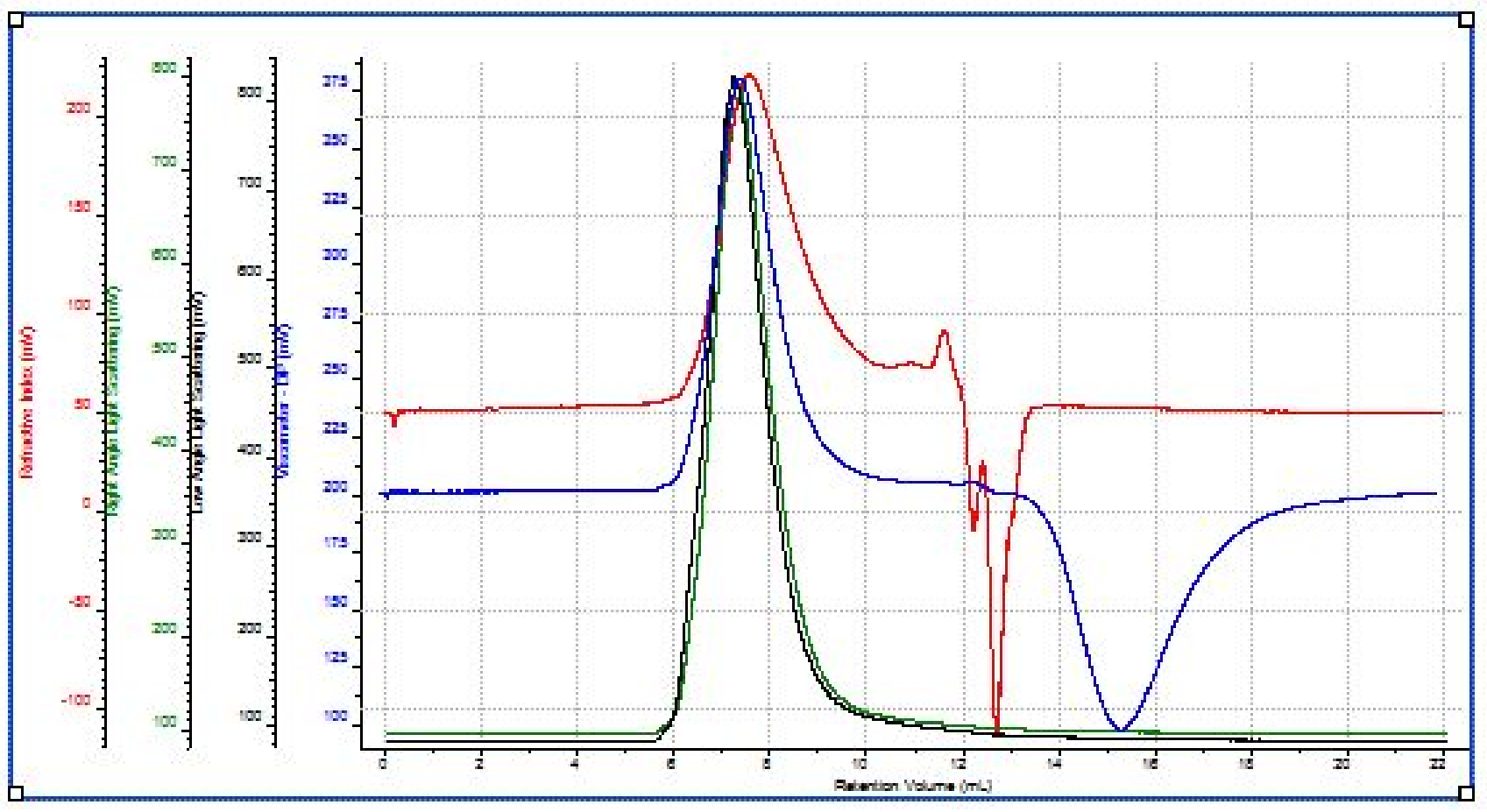

Figure S17. GPC chromatogram of APTCN-BPP 


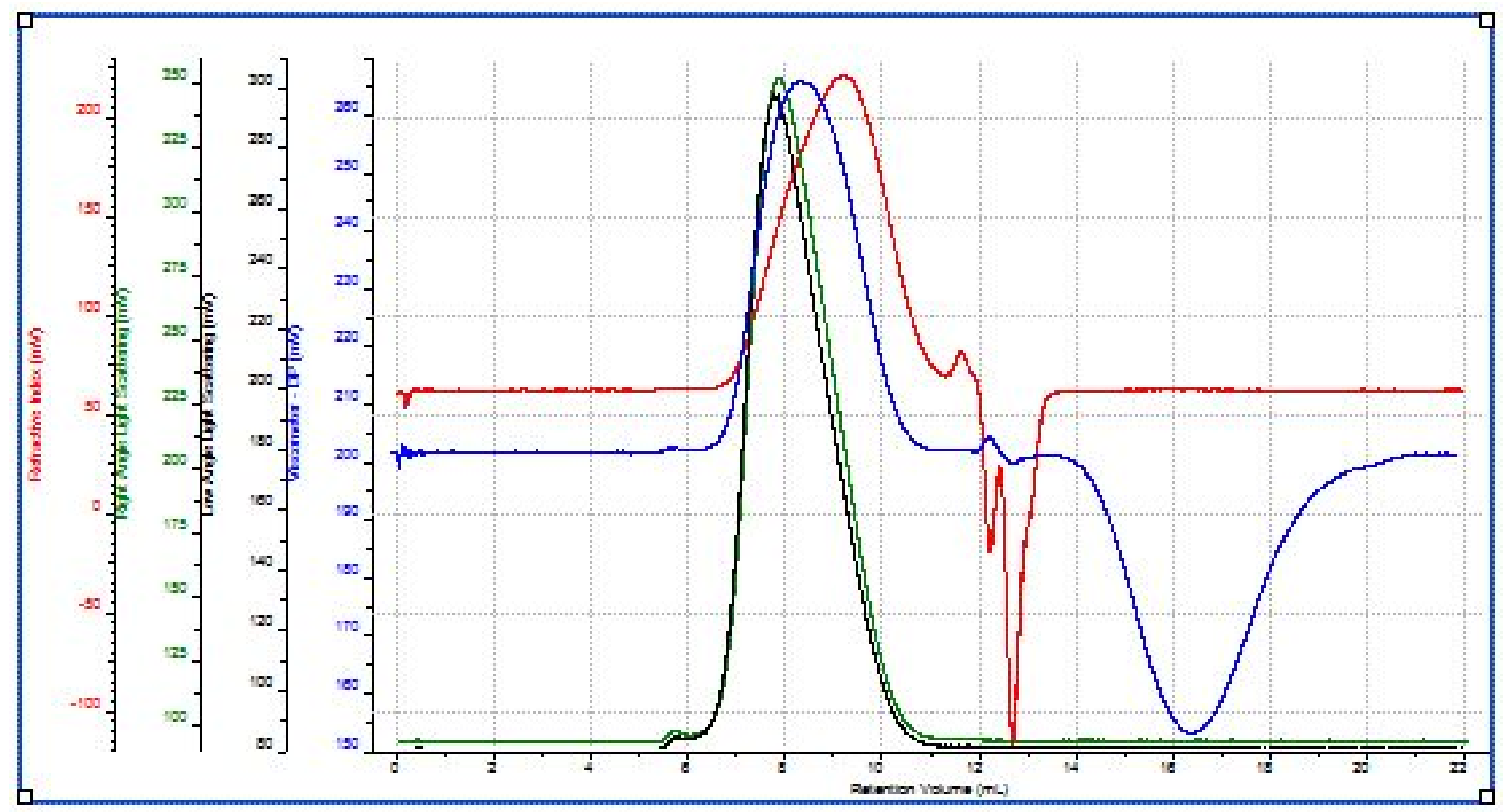

Figure S18. GPC chromatogram of APN-BPP

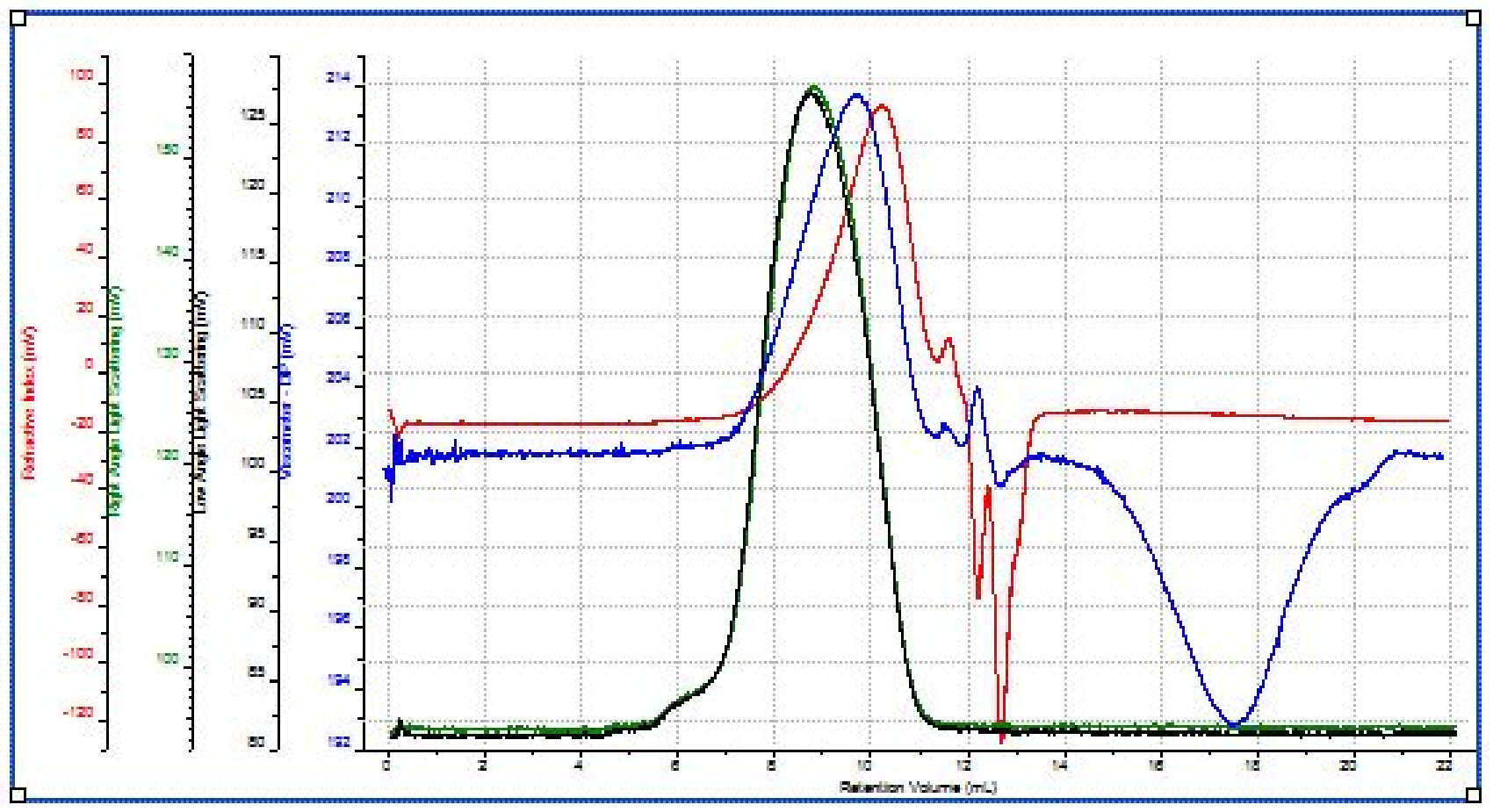

Figure S19. GPC chromatogram of ROMP-TCN-BPP 


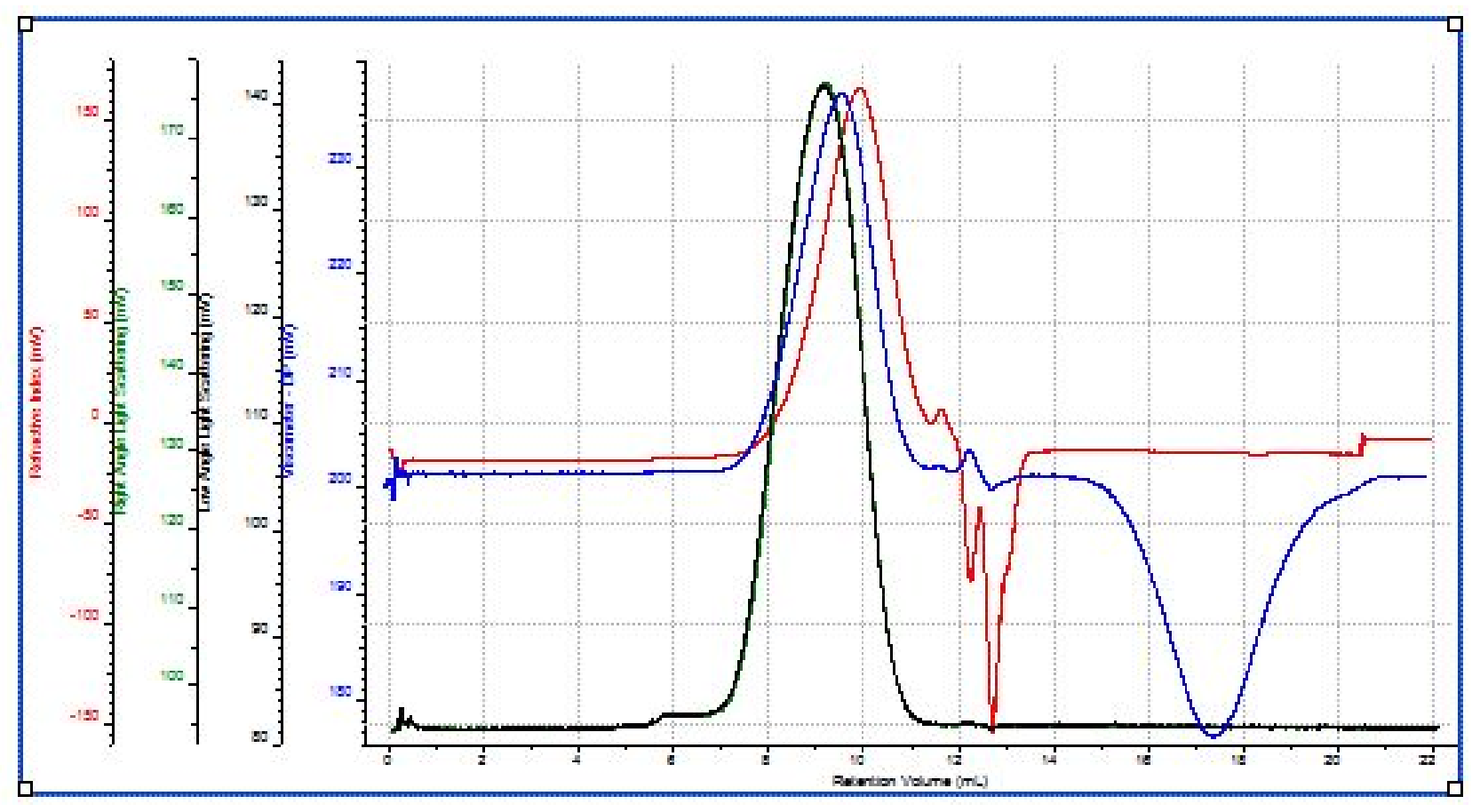

Figure S2o. GPC chromatogram of ROMP-N-BPP

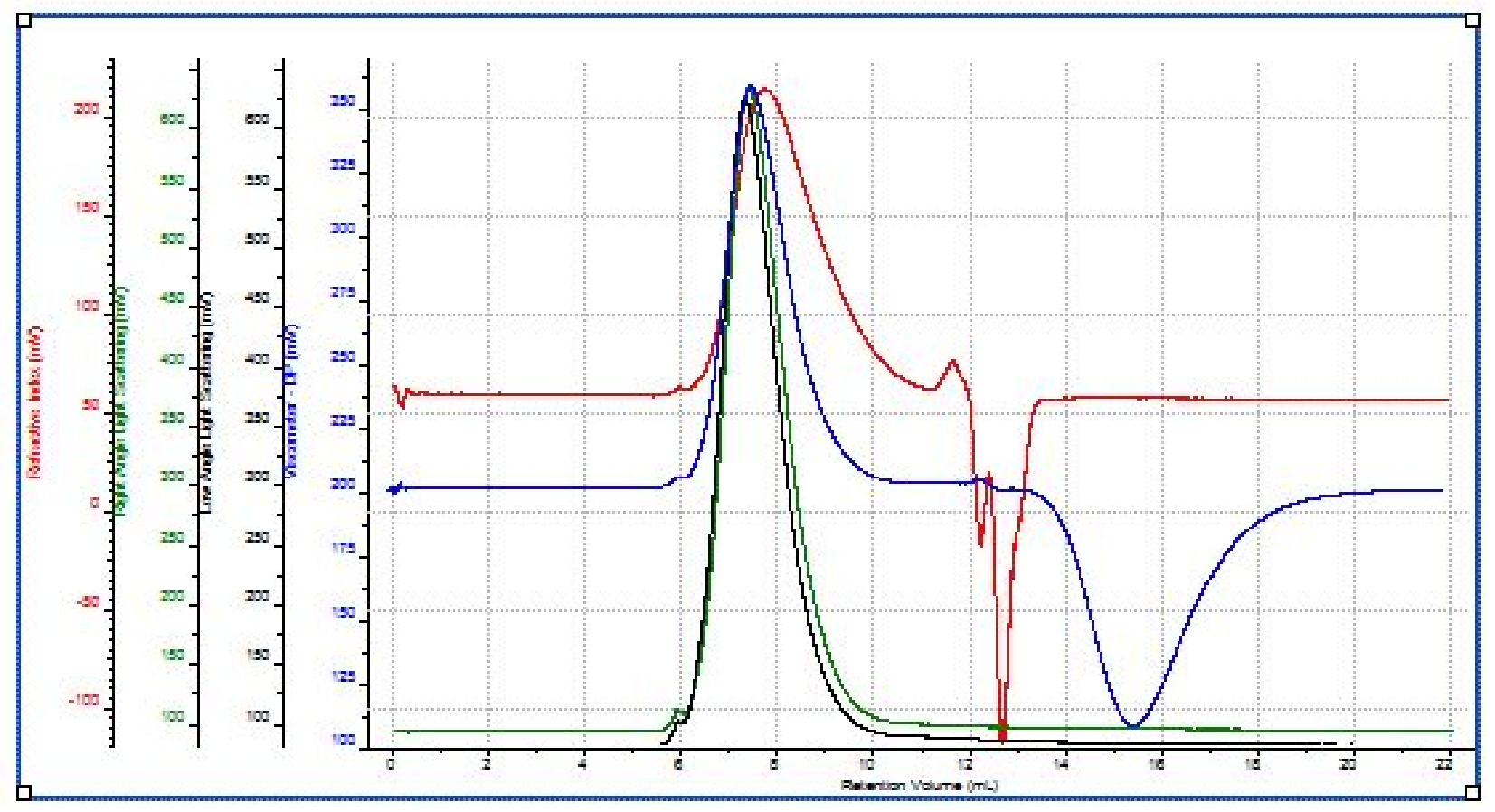

Figure S21. GPC chromatogram of APTCN-BPTMS 
Table S5. Molecular weight determinations for ROMP and addition-type polymers.

\begin{tabular}{lccc}
\cline { 2 - 4 } \multicolumn{1}{c}{ Polymer } & $\begin{array}{c}\mathrm{M}_{\mathrm{w}} \\
(\mathrm{kg} / \mathrm{mol})\end{array}$ & $\begin{array}{c}\mathrm{M}_{\mathrm{n}} \\
(\mathrm{kg} / \mathrm{mol})\end{array}$ & $\emptyset$ \\
\hline APTCN-BPP & 1,075 & 1,782 & 1.66 \\
APN-BPP & 267.6 & 720.3 & 2.69 \\
APTCN-BPTMS & 854.4 & 1,490 & 1.74 \\
ROMP-TCN-BPP & 89.67 & 353.8 & 3.93 \\
ROMP-N-BPP & 64.08 & 245.2 & 3.83 \\
\hline
\end{tabular}

Table S6. Simulated FFV comparisons show large FFV improvements in APTCN-exoBPTMS compared to APTCN-endo-BPTMS

\begin{tabular}{lllll}
\hline & $\begin{array}{l}\text { APTCN- } \\
\text { BPTMS }\end{array}$ & $\begin{array}{l}\text { APTCN- } \\
\text { BPP }\end{array}$ & $\begin{array}{l}\text { APN- } \\
\text { BPP }\end{array}$ & $\begin{array}{l}\text { APTCN- } \\
\text { endo-BPTMS }\end{array}$ \\
\hline$\rho$ (calcd., $\left.g / \mathrm{cm}^{3}\right)$ & 0.830 & 0.860 & 0.898 & 0.909 \\
& $( \pm 0.012)$ & $( \pm 0.017)$ & $( \pm 0.013)$ & $( \pm 0.007)$ \\
$F F V$ & 0.339 & 0.314 & 0.264 & 0.277 \\
& $( \pm 0.010)$ & $( \pm 0.013)$ & $( \pm 0.010)$ & $( \pm 0.005)$ \\
\hline
\end{tabular}
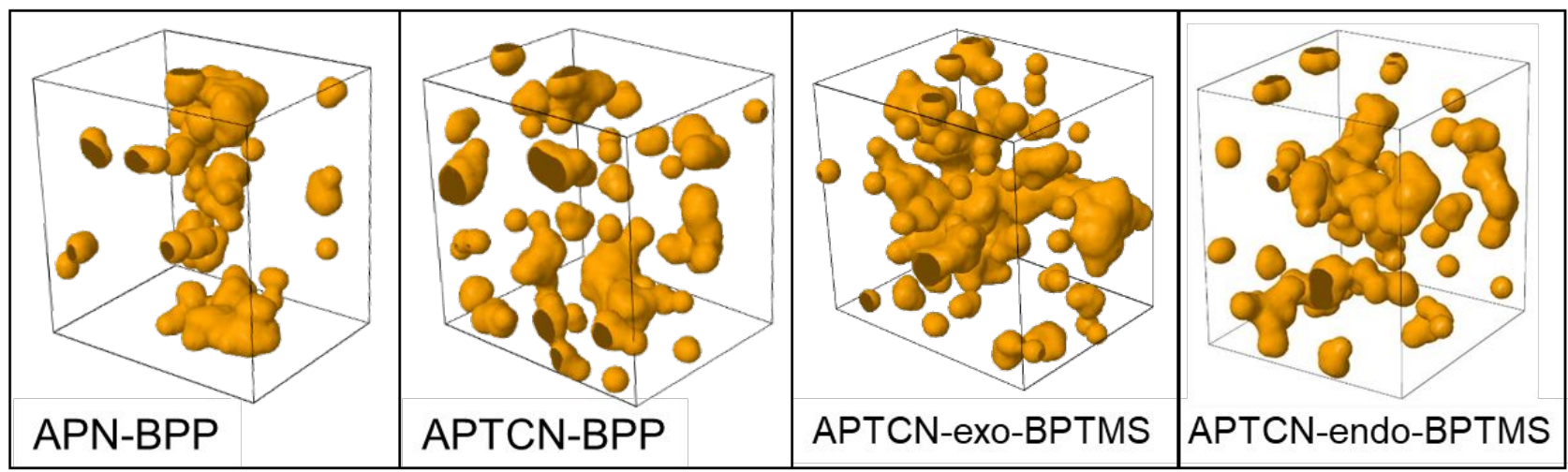

Figure S22. Simulated FFV comparison between APTCN-exo-BPTMS and APTCN-endoBPTMS. Modeled free volume available for butane permeation. The cavity isosurface plots use a probe radius equal to $2.15 \AA$, half the kinetic diameter of butane.

\section{References}

(1) Aida, F.; Sone, H.; Ogawa, R.; Hamaoka, T.; Shimizu, I. Palladium-catalyzed 5-exoSelective Reductive Mizoroki-Heck Reaction of Aryl Chlorides with 2,5-Norbornadiene. Chemistry Letters 2015, 44, 715. 